Equity Portfolio Diversification with High Frequency Data

Vitali ALEXEEV

University of Tasmania

Mardi DUNGEY

University of Tasmania

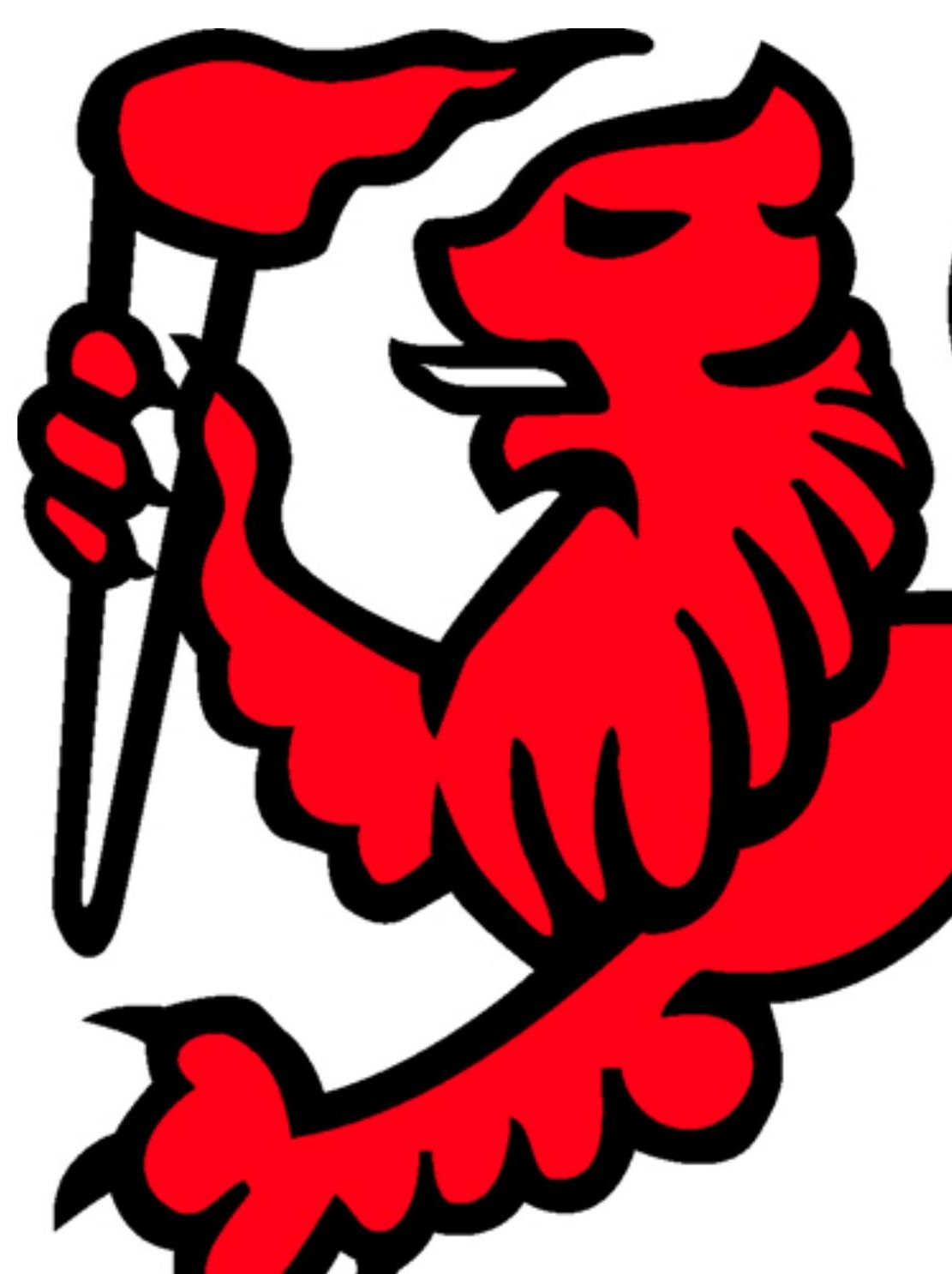




\title{
Equity portfolio diversification with high frequency data.
}

\author{
Vitali Alexeev ${ }^{\mathrm{a}, *}$, Mardi Dungey ${ }^{\mathrm{a}}$ \\ ${ }^{a}$ School of Economics and Finance, University of Tasmania, Hobart, Australia
}

\begin{abstract}
Investors wishing to achieve a particular level of diversification may be misled on how many stocks to hold in a portfolio by assessing the portfolio risk at different data frequencies. High frequency intradaily data provide better estimates of volatility, which translate to more accurate assessment of portfolio risk. Using 5-minute, daily and weekly data on S\&P500 constituents for the period from 2003 to 2011 we find that for an average investor wishing to diversify away 85\% (90\%) of the risk, equally weighted portfolios of 7 (10) stocks will suffice, irrespective of the data frequency used or the time period considered. However, to assure investors of a desired level of diversification $90 \%$ of the time, instead of on average, using low frequency data results in an exaggerated number of stocks in a portfolio when compared with the recommendation based on 5-minute data. This difference is magnified during periods when financial markets are in distress, as much as doubling during the 2007-2009 financial crisis.
\end{abstract}

Keywords: Portfolio diversification, high frequency, realized variance, realized correlation. JEL classification: G11, C63

Given the advantages of diversification, many experts recommend maximum diversification, also known as "buying the market portfolio." For an individual, constructing such a portfolio is difficult to say the least. Index funds that track market portfolios provide a good and less costly alternative. However, for actively managed funds, the large number of assets can result in elevated fund fees. If portfolio diversification can be achieved with a relatively small number of stocks, the need for funds comprising large numbers of assets might not be justified.

We trace the dynamics of the number of portfolio holdings, hereafter portfolio size, required to achieve a fixed level of diversification using 5-minute, daily and weekly data for the US equity market through the 2003-2011 period. Additionally, instead of assuming a fixed level of diversification, we fix the portfolio sizes at 5, 10, 20, 30 and 40 stocks and trace

\footnotetext{
*Corresponding author

Email addresses: valexeev@utas.edu.au (Vitali Alexeev), Mardi.Dungey@utas.edu.au (Mardi Dungey)
} 
the dynamics of diversification level through time. Early literature based on (semi-) annual and quarterly data and using standard deviation as a risk metric suggested that portfolios of 8 to 16 stocks are sufficient to achieve most of the available diversification benefits (Evans and Archer, 1968; Fisher and Lorie, 1970; Jennings, 1971; Fielitz, 1974; Johnson and Shannon, 1974). Subsequent work using monthly data supports a range of between 10 and 20 stocks (Klemkosky and Martin, 1975; Bloomfield, Leftwich, and Long, 1977; Bird and Tippett, 1986; Statman, 1987; Beck, Perfect, and Peterson, 1996; Brands and Gallagher, 2005 ). Solnik (1974) used weekly data for 8 international markets and found similar results. We are only aware of one study utilizing daily data to examine diversification; Domian, Louton, and Racine (2007). However, they estimate terminal wealth and terminal wealth standard deviation: measures independent of the data frequency. Although many studies investigate asset correlations using high frequency data and discuss its implications for portfolio diversification, e.g. Silvapulle and Granger (2001), we found no references to works directly exploring the benefits of using high frequency data for portfolio diversification.

High frequency data demonstrably improves estimation of risk. A range of efficient estimators has been developed offering a more accurate estimation of financial risk (see McAleer and Medeiros (2008) for an excellent survey on realized estimators), and in many applications high frequency data offers considerable gains to decision making. For example, realized volatility constructed from intraday data outperforms daily measures in forecasting future volatility (Blair, Poon, and Taylor, 2001; Andersen, Bollerslev, Christoffersen, and Diebold, 2006; Patton and Sheppard, 2009) and may improve hedging outcomes (Lai and Sheu, 2010). It has led to a considerable improvement in our understanding of how the data generating process of financial prices may be characterized (see particularly Aït-Sahalia and Jacod, 2012) and begun to improve our understanding of how price disruptions may be correlated across different assets and asset classes (see for example Dungey, McKenzie, and Smith, 2009; Todorov and Bollerslev, 2010). This approach is not without difficulties, however. Higher frequency sampling comes with the cost of microstructure noise, which can result in biased estimates. Although optimal sampling frequencies are the subject of ongoing research building from Bandi and Russell, 2006, currently a commonly accepted compromise in using high frequency data is to sample at 5 minute intervals (for example Wasserfallen and Zimmermann, 1985; Andersen and Bollerslev, 1997; Dacorogna, 2001; Hansen and Lunde, 2006; Lahaye, Laurent, and Neely, 2011).

This paper examines how the use of higher frequency data may affect recommendations for the number of stocks required to reduce risk to some pre-specified level. We compare results calculated using weekly, daily and 5 minute observations for equally weighted portfolios drawn from the S\&P500 constituent list over 2003 to 2011 for investors who wish to 
diversify $85 \%(90 \%)$ of risk. As a sample average it turns out that portfolios of $7(10)$ stocks will suffice irrespective of the frequency of price observation. However, tightening the risk reduction criteria so that the investor achieves the desired (85\% or 90\%) reduction in diversifiable risk in $90 \%$ of the time during the sample leads to quite different results. In this instance, examining higher frequency data allows us to dramatically reduce the number of stocks required to achieve the required risk reduction. Lower frequency sampling overstates the number of stocks required, and this is particularly evident during periods of market stress. Evidence from fixing the portfolio size vividly demonstrates that although during quiescent periods the difference in diversifiable risk assessed at different data frequencies is minimal, during periods of high volatility, when estimation of risk is key, the difference in diversifiable risk is quite pronounced. Using lower frequency data exaggerates estimates of diversifiable risk during periods of financial distress.

The paper proceeds as follows. Section I presents data and methodology, and Section II, presents the empirical results, followed by a conclusion in Section III.

\section{Data and Methodology}

Our data are obtained from Thomson Reuters Tick-History via SIRCA and consist of 5 minute intraday prices on constituents of the S\&P500 index from 2003 to 2011 during the trading day of 9:30 to 16:00 EST. Following convention, the intraday data are drawn as the last trade conducted during the 5 minute interval. The original data set consists of over 900 stocks from the constituent list of S\&P500 (RIC code \#0.SPX in the database). Only the stocks traded on NYSE and Nasdaq are retained; for details see Dungey, Luciani, and Veredas (2012). The choice of 5 minute data is consistent with the existing literature across a range of assets. The data do not consist of all stocks in the S\&P500 during the sample period, the data were selected to allow for a balanced panel in estimation subperiods, as discussed below, thus we do not totally account for survivorship bias. However, each of the measures we calculate, at differing frequency, face the same draw of companies. The sample contains 502 stocks over the sample period, and these are listed in a web appendix (attached to this submission for convenience).

Days in the sample are indexed by $t=1, \ldots, T$. Each day is divided into 5-minute intervals indexed by $i=0, \ldots, I$. The current price of an asset is then denoted by $S_{t, i}$, and the continuously compounded return $r_{t, i}$ is calculated as

$$
r_{t, i}= \begin{cases}\ln \left(\frac{S_{t, i}}{S_{t, i-1}}\right) & \text { for } i \geq 1 \\ \ln \left(\frac{S_{t, i}}{S_{t-1, I}}\right) & \text { for } i=0\end{cases}
$$


The second term in equation (1) represents the overnight return, and is deleted from the sample ${ }^{1}$, leading to a total number of return observations of $T \times I$. In order to match the volatility measures, daily observations are obtained based on the transaction price at the last grid-point time in day $t$. Similarly, weekly observations are obtained using the last grid-point time in each week. This ensures that identical transaction data are used for each frequency, and the only thing that we are changing is the length of the grid blocks. We use the same data set to get daily, $r_{t}^{(d)}$, and weekly, $r_{t}^{(w)}$, returns and define these below as

$$
\begin{aligned}
& r_{t}^{(d)}=\ln \left(\frac{S_{t, I}}{S_{t-1, I}}\right) \\
& r_{t}^{(w)}=\ln \left(\frac{S_{t, I}}{S_{t-5, I}}\right)
\end{aligned}
$$

Daily realized variance $(R V)$ is constructed as the sum of squared intraday returns

$$
R V_{(r), t}=\sum_{i=1}^{I} r_{t, i}^{2}
$$

and the average $R V$ for a period from $t=1$ to $T$ is found as

$$
\overline{R V}_{(r)}=\frac{1}{T} \sum_{t=1}^{T} R V_{(r), t}=\frac{1}{T} \sum_{t=1}^{T} \sum_{i=1}^{I} r_{t, i}^{2}
$$

Figure 1 provides some descriptive statistic on each of the S\&P500 constituents ranked by the magnitude of the average $R V$. Securities with high average $R V$ have lower average returns. Similar to Andersen, Bollerslev, Diebold, and Ebens (2001) we find that the unconditional distributions of realized variances are strongly right-skewed.

The corresponding measure of realized covariance, RCov, is expressed as

$$
\operatorname{Rov}_{\left(r_{1} r_{2}\right), t}=\sum_{i=1}^{I} r_{1, t, i}^{2} r_{2, t, i}^{2}
$$

and the average $R C o v$

$$
\overline{\operatorname{RCov}}_{\left(r_{1} r_{2}\right)}=\frac{1}{T} \sum_{t=1}^{T} \sum_{i=1}^{I} r_{1, t, i}^{2} r_{2, t, i}^{2} .
$$

Realized correlation on day $t$ is computed as

\footnotetext{
${ }^{1}$ We performed estimations inclusive of overnight returns and observed only slight change in our results with our main conclusion intact. Thus we omit these results from the paper for brevity but they are available upon request.
} 


$$
\rho_{\left(r_{1} r_{2}\right), t}=\frac{\sum_{i=1}^{I} r_{1, t, i}^{2} r_{2, t, i}^{2}}{\sqrt{\sum_{i=1}^{I} r_{1, t, i}^{2} \sum_{i=1}^{I} y_{t, i}^{2}}}=\frac{\operatorname{RCov}_{\left(r_{1} r_{2}\right), t}}{\sqrt{R V_{\left(r_{1}\right), t} R V_{\left(r_{2}\right), t}}} .
$$

The average realized correlation, $\bar{\rho}_{\left(r_{1} r_{2}\right)}$, for a period from $t=1$ to $T$ is found as

$\bar{\rho}_{\left(r_{1} r_{2}\right)}=\frac{1}{T} \sum_{t=1}^{T} \frac{\sum_{i=1}^{I} r_{1, t, i}^{2} r_{2, t, i}^{2}}{\sqrt{\sum_{i=1}^{I} r_{1, t, i}^{2} \sum_{i=1}^{I} r_{2, t, i}^{2}}} \neq \frac{\sum_{t=1}^{T} \sum_{i=1}^{I} r_{1, t, i}^{2} r_{2, t, i}^{2}}{\sqrt{\sum_{t=1}^{T} \sum_{i=1}^{I} r_{1, t, i}^{2} \sum_{t=1}^{T} \sum_{i=1}^{I} r_{2, t, i}^{2}}}=\frac{\overline{R C o v}_{\left(r_{1} r_{2}\right)}}{\sqrt{\overline{R V}_{\left(r_{1}\right)} \overline{R V}_{\left(r_{2}\right)}}}$

Note that the left-hand side and right-hand side of (9) are not equivalent when $T \neq 1$, and as $t$ increases the measures become more differentiated. Our methodology does not rely on the correlation estimates. Instead, we calculate unconditional correlations in Table 1 for comparison and illustrative purposes. This paper adopts the right hand side measure because we find it more appropriate to capture the average of daily measures for comparison with other metrics.

We construct portfolios by randomly drawing $n$ stocks without replacement from the entire sample in a particular time period. We use the same draw of stocks in estimation of risk metrics for each data frequency to avoid sample selection bias. Our portfolios are equally weighted to give a portfolio $P_{m}^{n}$, where $n=1 . . N$ indicates the number of stocks in the portfolio, $N$ is the total number of stocks available in the dataset during the subperiod analyzed, and $m=1 . . M$ represents the draw number. Given that our sample includes nonsurviving stocks, if a stock that is part of the chosen portfolio does not survive an initial period it is replaced with another randomly selected stock not already in the portfolio in the subsequent period.

We construct $M=5,000 n$-stock portfolios for each $n=1 . . N$, unless the number of combinations of $n$ stocks out of $N$ available is lower than $M$. For example, when $n=1$, the number of unique single security portfolios equals $N$ and when $n=N$ only one equally weighted portfolio can be constructed - we define it as the market portfolio. We find that 5,000 replications are sufficient to give a robust measure of central tendency of our risk measures.

For $n=1 . . N$ the return of the $n$-stock equally weighted random portfolio $m$ is defined as

$$
P_{m, \tau}^{n}=\sum_{j=1}^{n} \frac{\left\{r_{j, \tau}\right\}_{m}}{n}
$$

where $\tau$ is an equidistant time index defined as $\tau=1 . . T \times I$ for intraday returns, $\tau=1 . . T$ 
for daily and $\tau=1 . .\lfloor T / 5\rfloor$ for weekly. ${ }^{2}$

The average time series return over time of portfolio $m$ can be expressed as

$$
\bar{P}_{m}^{n}=\sum_{\tau} \frac{P_{m, \tau}^{n}}{\sup (\tau)}
$$

where $\sup (\tau)$ in the denominator of (11) allows us to refer to either the 5 minute, daily of weekly samples as appropriate. Let $\Omega_{m}^{n}$ represent a risk measure of an $n$-stock portfolio $m$. We define the average risk metric of $M$ portfolios, each of size $n$, as follows:

$$
\overline{\Omega^{n}}=\sum_{m=1}^{M} \frac{\Omega_{m}^{n}}{M}
$$

When equally weighted, the market portfolio consisting of all available securities is a unique portfolio, and $\overline{\Omega^{N}}=\Omega^{N}$. If $\overline{\Omega^{1}}$ and $\Omega^{N}$ are risk metrics for the average single-stock and market portfolios, we can define a scaled and standardized measure of diversification for an $n$-stock portfolio that adjusts for the average security risk and for the level of market risk. To derive the required number of securities for portfolios with a given level of diversifiable risk, we find it convenient to define a measure exclusively focused on diversifiable risk that is bounded from 0 to 1 as follows:

$$
\eta(n)=\frac{\overline{\Omega^{n}}-\Omega^{N}}{\overline{\Omega^{1}}-\Omega^{N}}
$$

We show the graphical representation of this measure in Figure 2 with a solid curve. Of course, the simplest way to express diversification is to plot the total risk against the portfolio size $\left(\overline{\Omega^{n}}\right.$ vs. $\left.n\right)$. Often, it is convenient to look at diversifiable risk only $\left(\overline{\Omega^{n}}-\Omega^{N}\right.$ vs. $n)$. However, when comparing multiple periods with different levels of total risk and nondiversifiable risk, it is best to standardize the diversification measure (as in equation 13 and as shown in Figure 2).

In addition, for a series of random draws of $n$-stock portfolios, let $\Omega_{q}^{n}$ be a $q$ th percentile of a risk measure $\Omega^{n}$. Similar to (13) we define:

$$
\eta(n, q)=\frac{\Omega_{q}^{n}-\Omega^{N}}{\overline{\Omega^{1}}-\Omega^{N}}
$$

and depict (14) in Figure 2 with a dashed curve.

Despite its drawbacks, standard deviation is most commonly used in the finance literature

\footnotetext{
${ }^{2}$ We recognize that aggregating log returns cross-sectionally is not the same as the log of aggregated simple returns, however the difference is small especially for 5 minute returns, and does not qualitatively affect our relative diversification measure in equation (13).
} 
as a measure of risk. The standard deviation of a portfolio is defined as follows:

$$
\Omega_{m}^{n} \equiv \sigma_{m}^{n}=\sqrt{\sum_{\tau} \frac{\left(P_{m, \tau}^{n}-\bar{P}_{m}^{n}\right)^{2}}{\sup (\tau)-1}}
$$

and the average standard deviation of $M$ random portfolios, each of size $n$ is

$$
\overline{\sigma^{n}}=\sum_{m=1}^{M} \frac{\sigma_{m}^{n}}{M}
$$

We derive the standard deviations based on daily data and weekly data. Average realized variance for the period is defined analogously to equation (5) as

$$
\Omega_{m}^{n} \equiv \overline{R V^{n}}=\sum_{m=1}^{M} \frac{R V_{m}^{n}}{M}
$$

Finally we require a choice of the target risk reduction via diversification. Our methodology assumes that portfolio total risk is comprised of systematic risk and specific or nonsystematic risk. As the number of securities included in a portfolio approaches the number of securities in the market, the portfolio risk approaches the overall level of systematic risk that is, market risk, suggesting a relationship which behaves as a decreasing asymptotic function. Reduction in portfolio risk can then be achieved up to the point where the incremental decrease in non-systematic risk brings insignificant benefits. Larger portfolios, however, are associated with higher transaction costs. We follow the existing literature which finds that an 85 to $95 \%$ reduction in risk via diversification is optimal (see Fisher and Lorie, 1970; Copp and Cleary, 1999; Kryzanowski and Singh, 2010 for empirical applications and discussions in Elton and Gruber, 1977; Tang, 2004 based on theoretical results). The next section presents results based on 85 and 90 percent risk reductions.

\section{Results}

Figures 3 and 4 depict the portfolio size needed to achieve desired level of risk reduction for an average investor and for investors requiring a particular level of diversification $90 \%$ of the time. For example, investors wishing to reduce the level of diversifiable risk in their portfolios by $85 \%$ (see Figure 3), require 7 stocks on average irrespective of the frequency

of the data or the time period analysed (Figure 3, solid lines). This is consistent with previous literature. Fisher and Lorie (1970) suggest portfolios of 8 to 16 stocks to achieve 85\% reduction, while Evans and Archer, 1968; Johnson and Shannon, 1974; Klemkosky and Martin, 1975 suggest portfolios comprising of as little as 3 to 10 stocks to achieve optimal diversification without specifying the exact percentage reduction in diversifiable risk. 
However, in the first part of our sample period (prior to financial crisis) to assure investors of the desired level of diversification $90 \%$ of the time instead of on average, the portfolio requirements suggest portfolios of 13-16 stocks when using daily data and 14-19 stocks when using weekly data (Figure 3, dashed lines). This is compared to portfolios of size 11-15 when using realized volatility as a risk measure with 5-minute data.

Figure 3 shows that there is considerable departure in the estimated numbers of stocks with different frequencies through time. In the earlier part of the sample, up until the second quarter of 2007, the disparities are not large. However, the advent of a period of financial stress, from the second half of 2007, and most particularly from third quarter of 2008 to first quarter 2009 associated with the collapse of Lehman Bros, rescue of AIG, the TARP program and complex negotiations concerning Bear-Stearns amongst other crisis events, changes this conclusion. While using realized variance as a risk measure, investors holding anywhere from 20 to 29 stocks can achieve $85 \%$ reduction in diversifiable risk $90 \%$ of the time, while the results using daily and weekly data suggest portfolios that at times are twice as large.

Figure 4 shows similar results for a $90 \%$ reduction of diversifiable risk. An average investor achieves $90 \%$ reduction in diversifiable risk with only $10-11$ stocks. This result is consistent across the risk measures and time periods and conforms to previous findings in the literature. Portfolio sizes required to achieve $90 \%$ reduction in diversifiable risk $90 \%$ of the times differ across measures and these difference is substantial during periods of market distress with larger recommended portfolio sizes when assessed with lower frequency data. ${ }^{3}$

Our results indicate that if the investor is concerned with reducing the diversifiable risk on average, then the choice among weekly, daily or 5-minute data will not have any impact on that decision. However, to assure the investor of the desired risk reduction level $90 \%$ of the time, using 5-minute data significantly lowers the number of stocks required in the portfolio. When the microstructure noise is removed, we argue that data with higher frequency provides a more accurate estimation of portfolio risk resulting in a lower confidence band around the average estimated portfolio size requirement. Using daily or weekly data frequency to arrive at the portfolio size recommendation may exaggerate and mislead investors wanting a particular degree of assurance $(85 \%$ or $90 \%$ of the time instead of achieving the set level of diversifiable risk reduction on average).

We admit that this reduction in portfolio size requirement might be subject to a number of important omissions, such as overnight trade and microstructure noise present in high frequency data. We have attempted to minimise these problems by avoiding the thin

\footnotetext{
${ }^{3}$ We conducted the same exercise but using median as our central tendency measure and the results are qualitatively similar and only marginally quantitatively different. The results are available upon request.
} 
overnight markets and concentrating on the constituents of S\&P500 only, which are heavily traded, instead of all securities listed on US national markets. Asymptotically, our derived diversification measure in equation 13 is quite appealing. It is unaffected by the inclusion or exclusion of overnight returns which makes it useful in a high frequency data setting. It is also unaffected by the scaling of the standard deviation or realized variance - which enables us to compare equivalently the sum of squared returns (typically used in realized variance measures) or the sum of squared demeaned returns (e.g., standard deviation). Although our results hold asymptotically, we also confirm empirically that this holds in our sample. These additional results are available upon request.

In Figure 5 instead of assuming a fixed level of diversification, we fix the portfolio at several size levels (i.e., 5, 10, 20, 30 and 40 stock portfolios). The left hand panel shows the percentage of diversifiable risk remaining in a portfolio of these fixed sizes calculated using daily data. The right hand panel gives the same measure but estimated using the 5-minute data. As expected, the diversifiable risk remaining in a portfolio reduces with the number of stocks. The levels of diversification are broadly consistent in both panels during the quiescent period (2003-early 2007). The crisis period (late 2007 - 2009), is characterised by a dramatic increase in the estimated diversifiable risk suggesting the need for larger portfolios, consistent with Figures 3 and 4. However, the diversification results from the 5 -minute data provide more reliable estimates since the underlining volatility measures are known to be more accurate when estimated using high frequency data (see Blair, Poon, and Taylor, 2001; Andersen, Bollerslev, Christoffersen, and Diebold, 2006; Patton and Sheppard, 2009). The heightened level of diversifiable risk indicated with the daily data suggest excessively large portfolios, sometimes more than doubling the number of stocks held suggested by the 5-minute estimates. Working with an even lower frequency data, such as monthly, is only likely to exacerbate this problem.

The availability of a large number of observations, as in the case of high frequency data, enables us to estimate extreme tail risk measures without the need for model-based bootstrap techniques, which may suffer from model estimation biases. Thus relying on historical observations only, we are able to estimate expected shortfall measures at 95\%, 99\% and 99.9 \% levels and reconstruct in Figure 6 the recommended portfolio sizes to achieve $90 \%$ reduction in diversifiable risk along with the confidence bands needed to assure this reduction $90 \%$ of the time. These results are largely consistent with those obtained from Figures 3 and 4. An interesting feature is that as the risk measure becomes more extreme, the recommended portfolio size decreases independently of market conditions. This aspect is worthy of a future separate investigation.

The results in Figures 3 and 4 strongly indicate the difference in portfolio size recom- 
mendations during tranquil vs distressed markets. To explore this further we implement the conditional correlation analysis of Silvapulle and Granger (2001) who consider the differences between bear and bull markets. We estimate conditional correlations for portfolios for the period from 2003 to 2011 using one month rolling window with a 12 month estimation period at each estimation point. As we have 502 assets in the data sample, this involves drawing a possible 125,751 combinations of stocks. As in Silvapulle and Granger (2001) we conduct quantile analysis, and concentrate on the upper and lower $5 \%$ tails, while presenting also the results for the 'middle' quantile between them. Thus, we anticipate that there may well be fewer than 125,751 combinations in the upper and lower quantiles when particular stocks do not have tail events.

Table 1 shows the average unconditional correlations for each of the full sample and the pre-crisis period prior to July 2007 and the period thereafter, and conditional correlation coefficients for the three quantiles for the same subsamples. The unconditional results show that during the pre-crisis subsample correlation is lower than in the second half of the sample. This increase in unconditional correlation coefficients between a period of tranquility and a period of stress is well recognised in the literature as a sign of stress, for example Butler and Joaquin (2002), although it is necessary to account for the possibility that the observed result may be a simple consequence of increased volatility rather than a sign of distressed conditions; see Forbes and Rigobon (2002). It is clear that the increases in conditional correlation for the middle quantile is less dramatic. However, the increases in correlation for the tail returns is much more pronounced, particularly in the case of the upper tail.

We now examine these characteristics in more detail. The results for the three quantiles are presented in Figure 7 for a 12 month estimation window. The solid line represents the average conditional correlation for the medium quantile with the solid shaded area representing the interquantile range around it. Each point on the graph represents the average conditional correlation calculated from stock returns for the previous and following 6 months, that is the point is the centre of the rolling sample. It is quite clear that the average of the conditional correlations for this medium quantile shows an upward move from values of 0.15 or below prior to the crisis period in mid-2007 and rises steadily to peak in early 2009, consistent with a period of calculation which encompasses the volatile second half of 2008 and the first half of 2009 before the presumed end of the US recession according to NBER dating.

The second rise in the average conditional correlation relates to increased international financial volatility associated with the burgeoning European debt crisis - visible first in Greece in early 2010 (and thus represented in the figure from mid-2009 onwards due to the data centering) and the further escalation of this crisis in 2011. The figure is completely consistent with an analysis of generally rising volatility conditions in financial markets during the crisis 
period, which will result in rising correlation even without changes in the interrelationships between assets. The pronounced rise in correlation of the stocks in the bear market associated with the crisis originating in the US is consistent with many papers on the poor performance of diversification during periods of stress; for an example in non-equity markets see Knight, Lizieri, and Satchell (2005).

The upper and lower quantiles present interesting variations on the middle quantile result. Up until the crisis period the tail quantiles were not particularly different in profile to the middle quantile. As we would expect their interquantile range is larger, but the point estimates are sometimes below and sometimes above that for the middle quantile. However, in periods of stress the tail quantiles experience a much more notable rise in correlation than the middle quantile - thus the rise in volatility across different assets is demonstrably not the same, lending credence to this measure as representing a change in market interactions not associated with generally increased market volatility. The marked increase in correlations of tail returns during periods of stress was also noted for the lower tail by Silvapulle and Granger (2001).

The difference in lower and higher quantiles in Figure 7 shows two major periods of increase in correlation. These are the period around the US financial crisis, beginning in mid-2008 (and thus visible in the 12 month centred data for the observation around March 2008), when the average correlation in the upper tail rose more than the average correlation in the lower tail. This is consistent with a domestically sourced crisis which corresponded to a domestic recession associated with both flight out of stocks generally, and flight towards relatively higher performing (blue-chip) stocks within the market.

During the rise in correlation associated with the European sovereign debt problems the tail correlations rose more than in the US based crisis period, and the correlation in the lower tail rose more than in the upper tail. In this period the US economy is recovering, albeit slowly, and the outlook for US equity markets is more positive. The finding that the lower tail quantiles are generally more correlated than the middle quantiles is consistent with the existing findings on the behavior of poorly performing stocks; Butler and Joaquin (2002), Silvapulle and Granger (2001). The higher correlation amongst the lower performing stocks may well represent the sluggish nature of the economy in some sectors, particularly if sectors of the economy exhibit differing behaviours.

To investigate the behavior of the lower tails more carefully we conduct the same analysis using the 12 month estimation windows for 9 sectors of the economy; materials, congolmerates, consumer goods, finance, health care, industrials, technology, services and utilities. The results shown in Figure 8 are striking. In almost all cases, during the period associated with the US based crisis and US recession the correlation of the higher performing stocks is greater 
than the correlation of the lowest performing stocks, but for the materials, conglomerates and technology sectors the lower tail correlations are below those of the middle quantile they are reacting less than the market to the stressful conditions. The one exception is for consumer goods, where the two tails have an approximately equal rise in correlation, potentially reflecting the sluggish return of consumption in the US recovery. The largest gap between the correlations of the upper and lower tails during the 2010-11 period is for the health care sector.

In the financial sector results, correlations amongst both the highest performing stocks and the lowest performing stocks are below those of the middle quantile stocks. Although this may at first appear counterintuitive the results are consistent with the events. The highest performing stocks in this sector were very diverse as the sector was in complete dissarray - and insurance and banking sector stocks often provided completely different pictures as different support packages and bailouts were announced. Dungey, Luciani, and Veredas (2012) provide a detailed analysis. In the period from 2010, when the European crisis became the dominant concern of international financial markets all sectors of the US market experienced higher correlation amongst the lowest performing stocks than the highest performing tail. This aligns with the usual findings for bear markets, that the lowest performing stocks in the sector are most vulnerable to loss of investor confidence.

\section{Conclusion}

We find that for investors wishing to diversify away $85 \%$ (90\%) of the risk, equally weighted portfolios of 7 (10) stocks will suffice irrespective of the data frequency used or the time period considered. However, to assure the investors of the desired level of diversification $90 \%$ of the time, the portfolio requirements based on lower frequency data are exaggerated when compared with the results based on the 5-minute data. We find that this difference is greater during the periods when financial markets are in distress. Assuming risk measures based on higher frequency data are superior to their lower frequency based counterparts, investors may not need to hold portfolios as large as otherwise suggested by lower frequency risk measures, especially during financial crisis episodes.

The high frequency data allow us to assess conditional correlations between stocks for moving windows during the sample period. We ascertain that the changes in the correlation between stocks occur during periods of stress, generally increases, but that this is particularly the case for upper and lower tail performing stocks. During the US based crisis period, associated with a domestic recession, correlation amongst the best performing stocks increased more than that between the worst performing stocks. However, during the later period in 2010-11 where the US was recovering and international financial markets were stressed 
by international events originating in Europe, correlation between the lowest performing stocks exceeded that of the worst performing stocks, consistent with existing literature that domestic stocks behave differently when highly correlated with an international bear market.

\section{References}

Andersen, Torben G., and Tim Bollerslev, 1997, Intraday periodicity and volatility persistence in financial markets, Journal of Empirical Finance 4, 115 - 158.

— , Peter F. Christoffersen, and Francis X. Diebold, 2006, Chapter 15 volatility and correlation forecasting, in C.W.J. Granger G. Elliott, and A. Timmermann, ed.: Handbook of Economic Forecasting vol. 1 . pp. 777-878 (Elsevier).

Andersen, Torben G., Tim Bollerslev, Francis X. Diebold, and Heiko Ebens, 2001, The distribution of realized stock return volatility, Journal of Financial Economics 61, 43 76.

Ait-Sahalia, Yacine, and Jean Jacod, 2012, Analyzing the spectrum of asset returns: Jump and volatility components in high frequency data, Journal of Economic Literature 50, $1007-1050$.

Bandi, F. M., and J. R. Russell, 2006, Separating microstructure noise from volatility, Journal of Financial Economics 79, 655-692.

Beck, Kristine L., Sieven B. Perfect, and Pamela P. Peterson, 1996, The role of alternative methodology on the relation between portfolio size and diversification, Financial Review $31,381-406$.

Bird, Ron, and Mark Tippett, 1986, Naive diversification and portfolio risk-a note, Management Science 32, pp. 244-251.

Blair, Bevan J., Ser-Huang Poon, and Stephen J. Taylor, 2001, Forecasting S\&P 100 volatility: The incremental content of implied volatilities and high-frequency index returns., Journal of Econometrics 105, 5-26.

Bloomfield, Ted, Richard Leftwich, and John B. Jr. Long, 1977, Portfolio strategies and performance, Journal of Financial Economics 5, 201 - 218.

Brands, Simone, and David R. Gallagher, 2005, Portfolio selection, diversification and fundof-funds: a note, Accounting $\&$ Finance 45, 185-197. 
Butler, K.C., and D.C. Joaquin, 2002, Are the gains from international portfolio diversification exaggerated? The influence of downside risk in bear markets, Journal of International Money and Finance 21, 981-1011.

Copp, David, and Sean Cleary, 1999, Diversification with Canadian stocks: How much is enough?, Canadian Investment Review 12, 21-25.

Dacorogna, M., Gencay R. Müller U. Olsen R. B. Pictet O. V., 2001, An Introduction to High Frequency Finance (London: Academic Press).

Domian, Dale L., David A. Louton, and Marie D. Racine, 2007, Diversification in portfolios of individual stocks: 100 stocks are not enough, Financial Review 42, 557-570.

Dungey, M., M. Luciani, and D. Veredas, 2012, Ranking systemically important financial institutions, manuscript.

Dungey, Mardi, Michael McKenzie, and L. Vanessa Smith, 2009, Empirical evidence on jumps in the term structure of the US Treasury market, Journal of Empirical Finance 16, $430-445$.

Elton, Edwin J., and Martin J. Gruber, 1977, Risk reduction and portfolio size: An analytical solution, The Journal of Business 50, pp. 415-437.

Evans, John L., and Stephen H. Archer, 1968, Diversification and the reduction of dispersion: An empirical analysis, The Journal of Finance 23, pp. 761-767.

Fielitz, Bruce D., 1974, Indirect versus direct diversification, Financial Management 3, pp. $54-62$.

Fisher, Lawrence, and James H. Lorie, 1970, Some studies of variability of returns on investments in common stocks, The Journal of Business 43, pp. 99-134.

Forbes, K., and R. Rigobon, 2002, No contagion, only interdependence: Measuring stock market co-movements., Journal of Finance 57, 2223-2261.

Hansen, Peter R., and Asger Lunde, 2006, Realized variance and market microstructure noise, Journal of Business \&3 Economic Statistics 24, pp. 127-161.

Jennings, Edward H., 1971, An empirical analysis of some aspects of common stock diversification, Journal of Financial and Quantitative Analysis 6, 797-813.

Johnson, Keith H., and Donald S. Shannon, 1974, A note on diversification and the reduction of dispersion, Journal of Financial Economics 1, 365 - 372. 
Klemkosky, Robert C., and John D. Martin, 1975, The effect of market risk on portfolio diversification, The Journal of Finance 30, pp. 147-154.

Knight, John, Colin Lizieri, and Stephen Satchell, 2005, Diversification when it hurts? The joint distributions of real estate and equity markets, Journal of Property Research 22, 309-323.

Kryzanowski, Lawrence, and Singh Singh, 2010, Should minimum portfolio sizes be prescribed for achieving sufficiently well-diversified equity portfolios?, Frontiers in Finance and Economics 7, 1-37.

Lahaye, L., S. Laurent, and C. Neely, 2011, Jumps, cojumps and macro announcements., Journal of Applied Econometrics 26, 893-921.

Lai, Y.-S., and H.-J. Sheu, 2010, The incremental value of a futures hedge using realized volatility, Journal of Futures Markets 30, 874-896.

McAleer, Michael, and Marcelo Medeiros, 2008, Realized volatility: A review, Econometric Reviews 27, 10-45.

Patton, A., and K. Sheppard, 2009, Optimal combinations of realised volatility estimators, International Journal of Forecasting 25, 218-238.

Silvapulle, P., and C.W.J. Granger, 2001, Large returns, conditional correlation and portfolio diversification: a value-at-risk approach, Quantitative Finance 1, 542-551.

Solnik, Bruno H., 1974, Why not diversify internationally rather than domestically?, Financial Analysts Journal 30, pp. 48-52+54.

Statman, Meir, 1987, How many stocks make a diversified portfolio?, The Journal of Financial and Quantitative Analysis 22, pp. 353-363.

Tang, Gordon Y.N., 2004, How efficient is naive portfolio diversification? An educational note, Omega 32, 155 - 160.

Todorov, V., and T. Bollerslev, 2010, Jumps and betas: A new framework for estimating and disentangling systematic risk., Journal of Econometrics 157, 220-235.

Wasserfallen, Walter, and Heinz Zimmermann, 1985, The behavior of intra-daily exchange rates, Journal of Banking \& Finance 9, 55-72. 

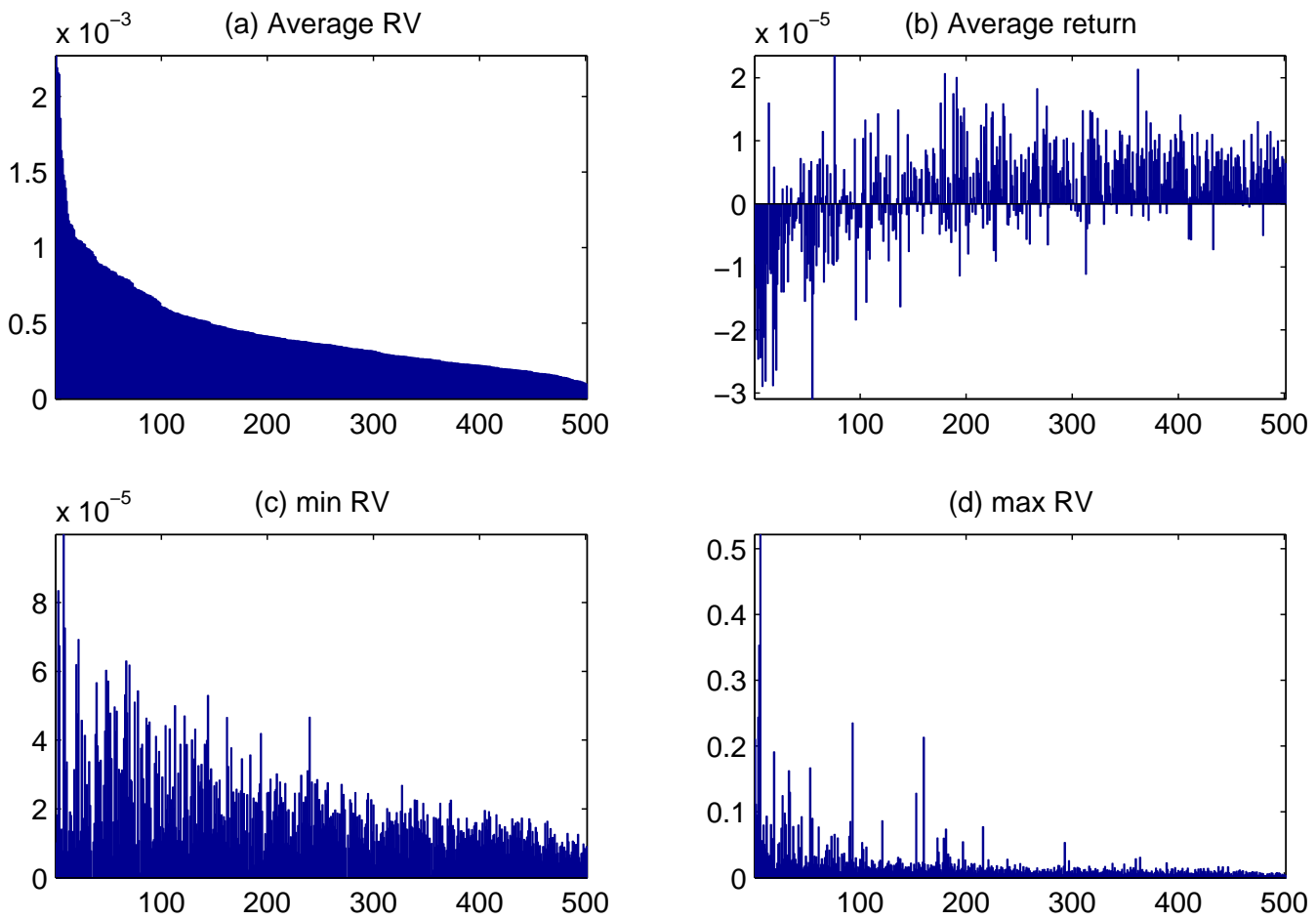

(d) $\max R V$

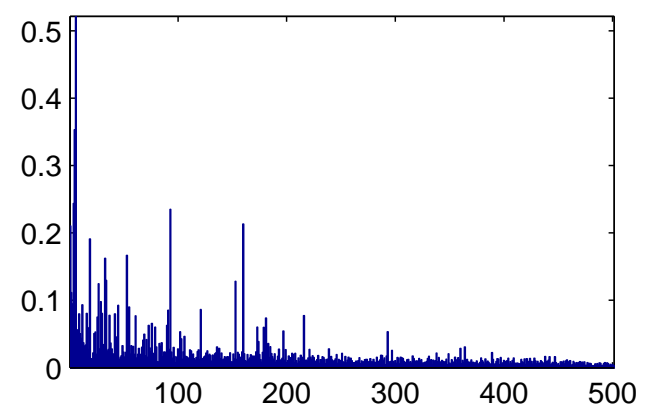

(e) Skewness RV

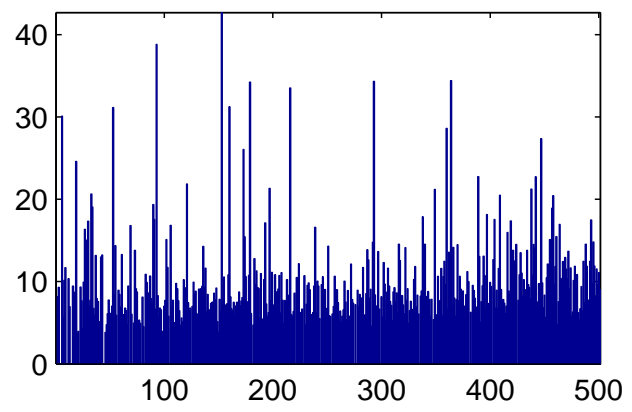

(f) Kurtosis RV

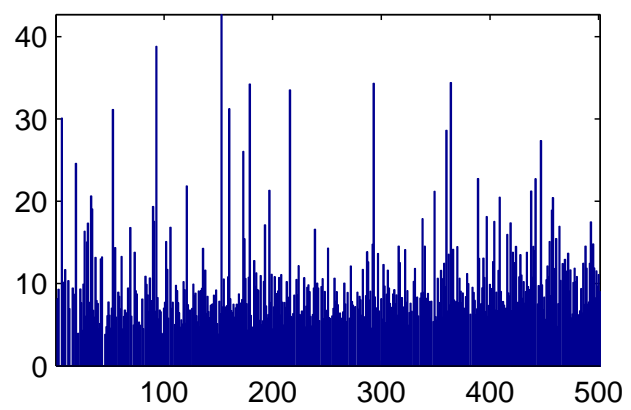

Figure 1: Descriptive Statistic. The horizontal axis represents the index for each of the S\&P 500 constituents ranked by the magnitude of the average RV. Similar to Andersen, Bollerslev, Diebold, and Ebens (2001) we find that the unconditional distributions of realized variances are highly right-skewed. 


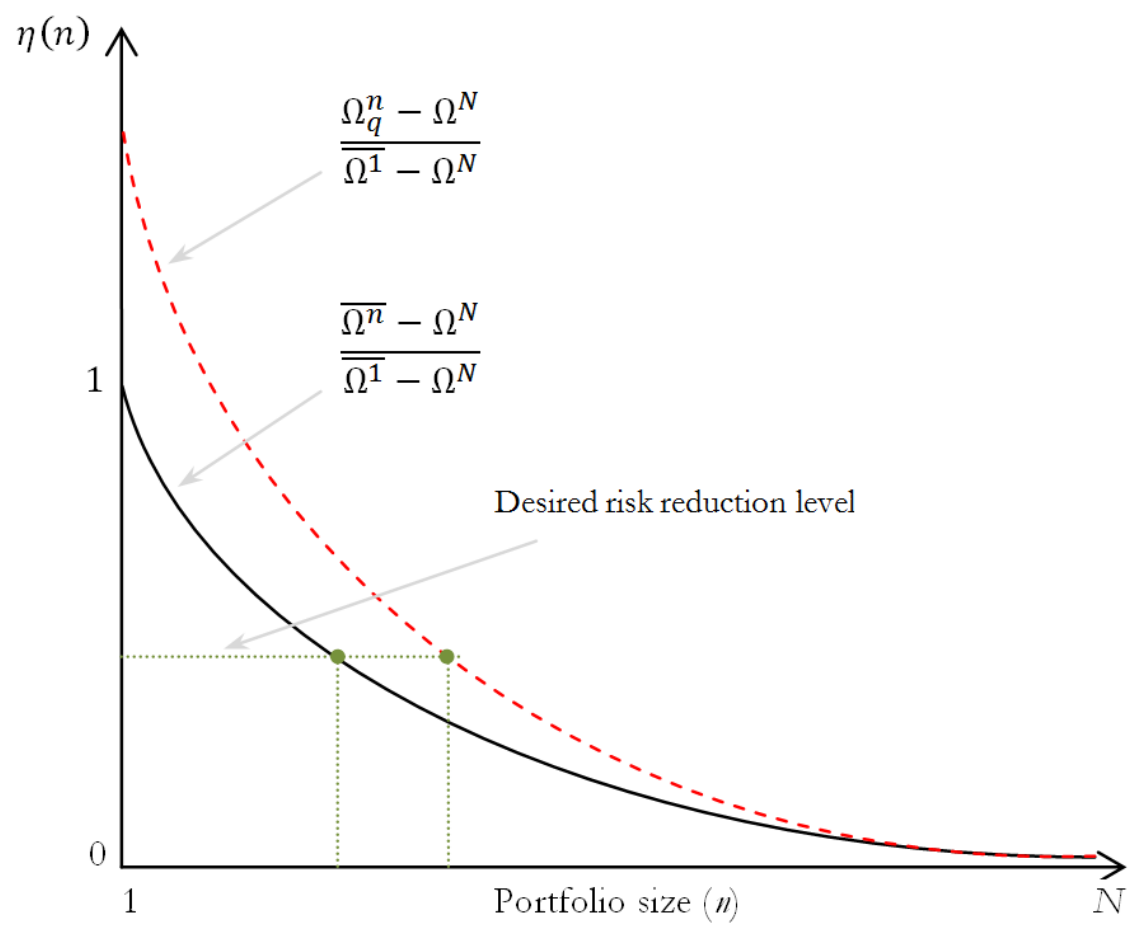

Figure 2: STANDARDIZED RISK AS A FUnCTION OF PORTFOlio SIZE. The solid black curve represents the average standardized risk measure as defined in (12). The dashed red curve represents the $q$ th percentile of the standardized risk measure and is defined in (13). 
SP500: Recommended portfolio size to achieve $85 \%$ reduction in diversifiable risk.

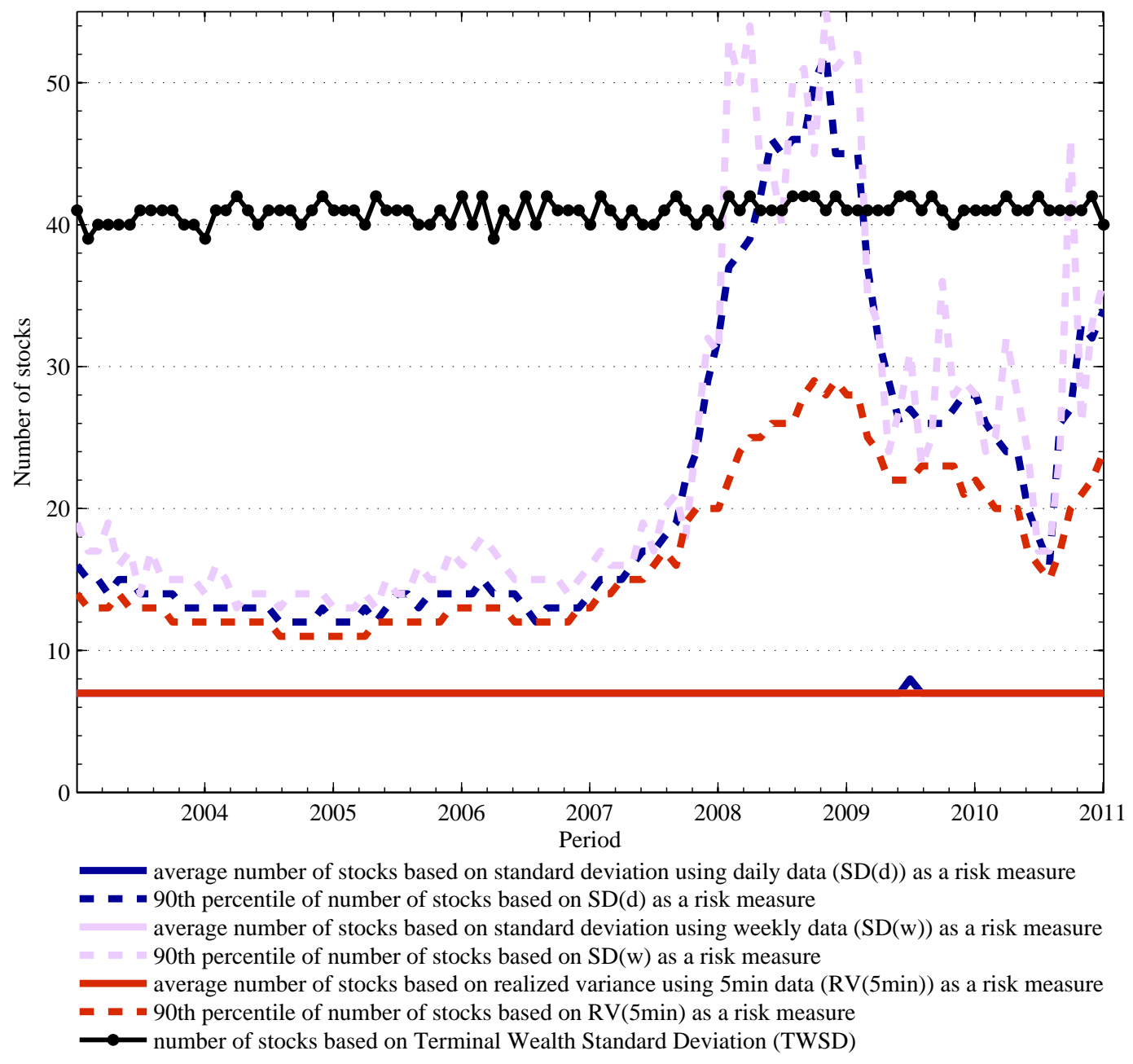

Figure 3: Portfolio Size REQUiREMEnT FOR 85\% REDUCTION IN DIVERSIFIABLE RISK. The figure depicts portfolio size recommendations through time and across three different risk measure based on 5-minute, daily and weekly frequencies. We use realized variance as a measure of risk using 5-minute data, standard deviation with daily and weekly data. We have also derived recommendations based on sum of squared returns using daily and weekly data to make it equivalent to realized volatility. Our results were identical to the ones obtained from standard deviation for both weekly and daily. Portfolio size recommendations based on risk measure with higher frequency data are generally smaller. The difference is minimal during the normal market conditions and exacerbated during the periods of market distress. To obtain the results we repeat the analysis every month using one year of past data. 
SP500: Recommended portfolio size to achieve $90 \%$ reduction in diversifiable risk.

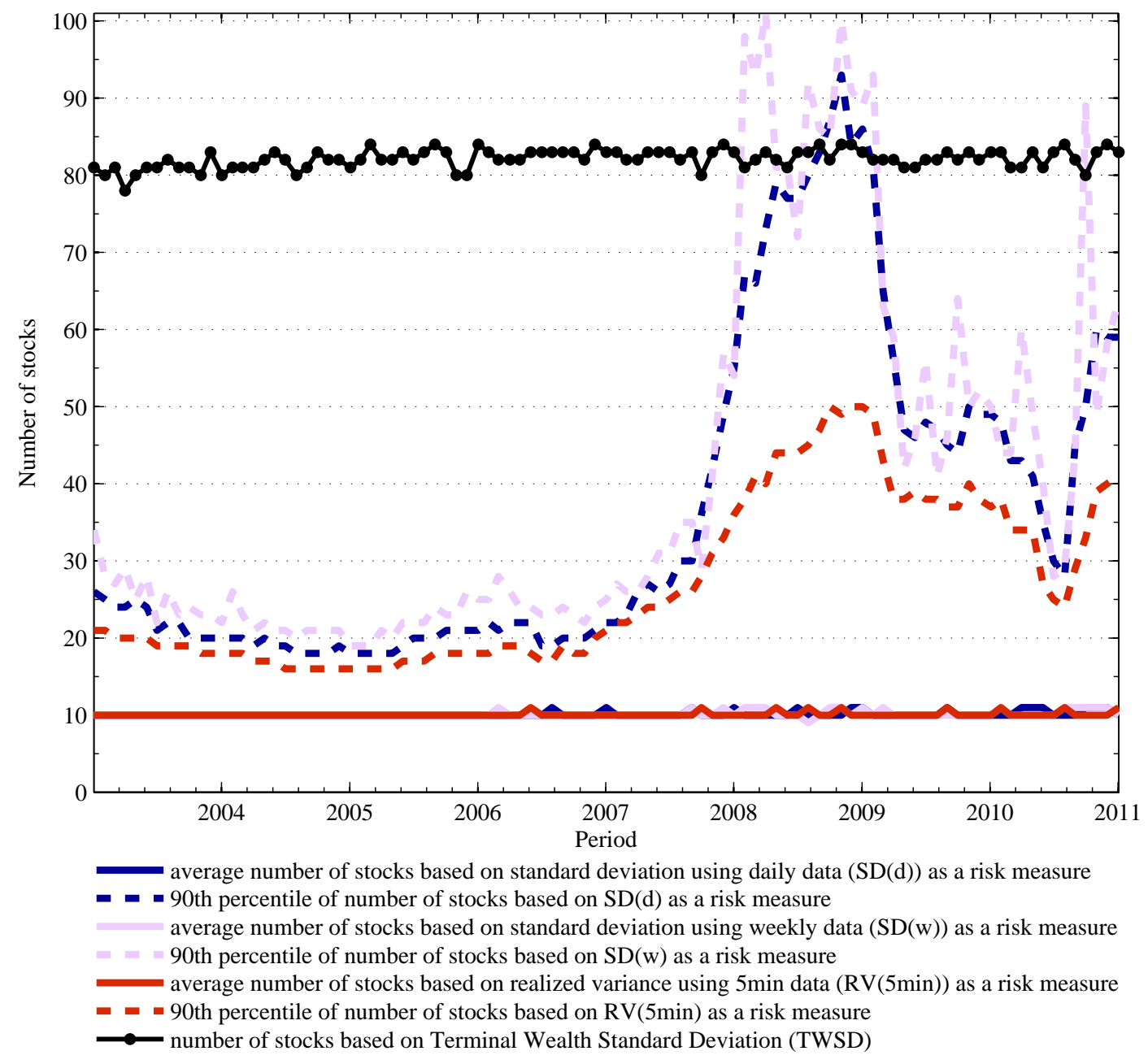

Figure 4: Portfolio size ReQUiREment For 90\% REDUCtion IN DIVERSifiable Risk. The figure depicts portfolio size recommendations through time and across three different risk measure based on 5-minute, daily and weekly frequencies. We use realized variance as a measure of risk using 5-minute data, standard deviation with daily and weekly data. We have also derived recommendations based on sum of squared returns using daily and weekly data to make it equivalent to realized volatility. Our results were identical to the ones obtained from standard deviation for both weekly and daily. Portfolio size recommendations based on risk measure with higher frequency data are generally smaller. The difference is minimal during the normal market conditions and exacerbated during the periods of market distress. To obtain the results we repeat the analysis every month using one year of past data. 

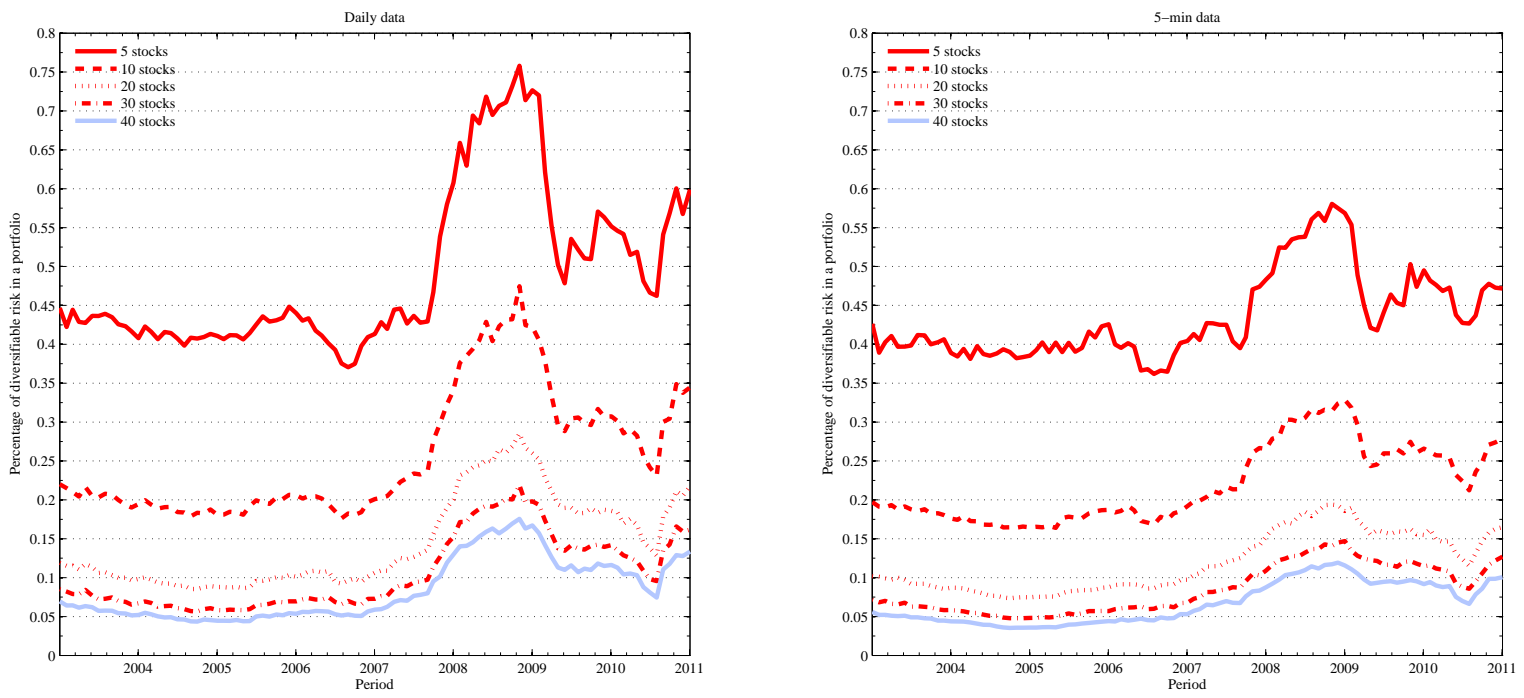

Figure 5: Diversifiable RISK REMAINING FOR PORTFOlios OF VARIOUS SIZES. As the number of stocks in portfolios increases the percentage of diversifiable risk decrease changes over the years. The panels above show the dynamics of diversifiable risk remaining for portfolios of various sizes. Results are obtained for an investor seeking to diversify with assurance $90 \%$ of the time. The left panel is based on standard deviations with daily data; the panel on the right uses 5-minute data and is based on the realized volatility. 
SP500: Recommended portfolio size to achieve $90 \%$ reduction in diversifiable risk.

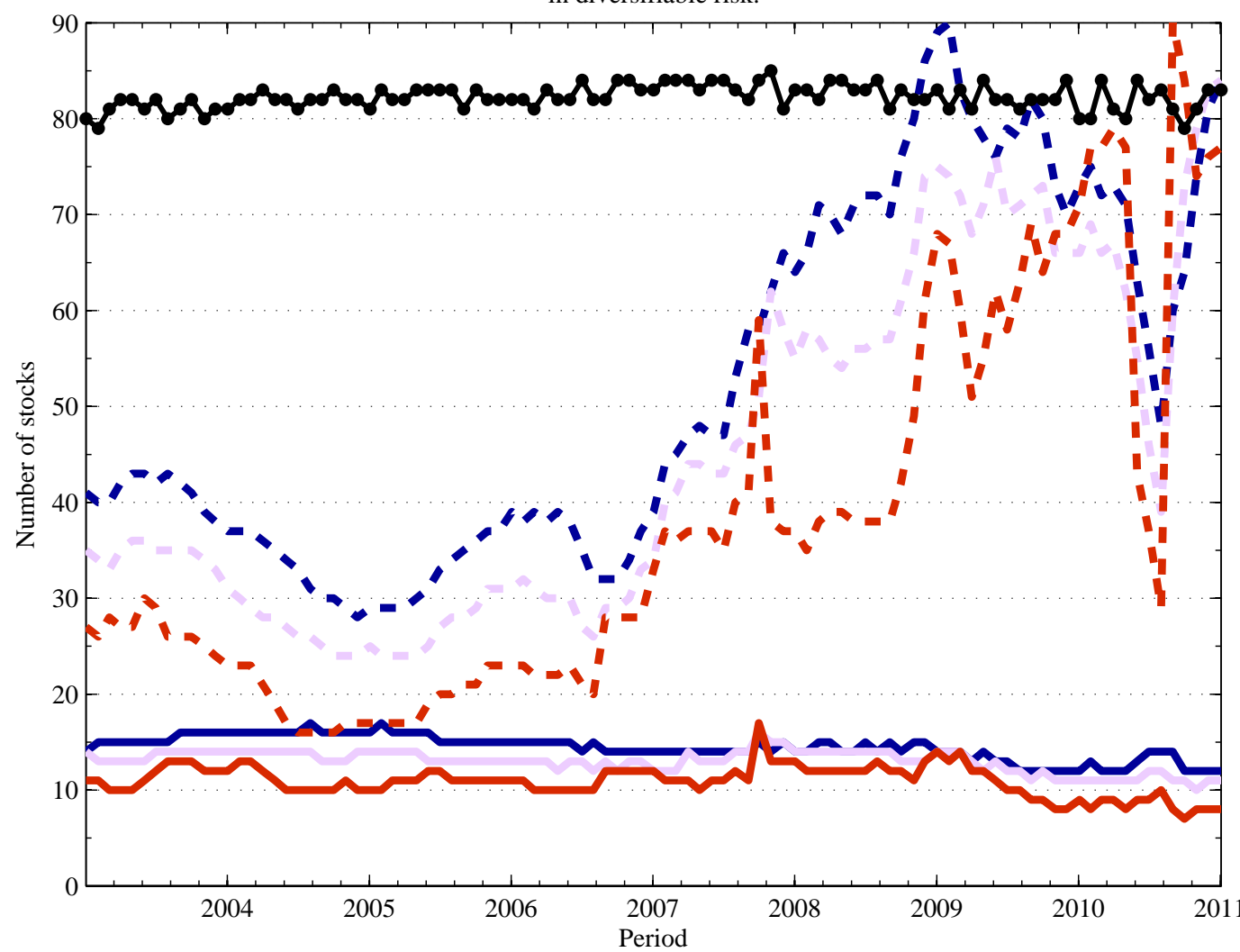

average number of stocks based on Expected Shortfall at 95\% (ES(95\%)) as a risk measure

- - 90th percentile of number of stocks based on ES(95\%) as a risk measure average number of stocks based on Expected Shortfall at 99\% (ES(99\%)) as a risk measure 90th percentile of number of stocks based on $\mathrm{ES}(99 \%)$ as a risk measure

average number of stocks based on Expected Shortfall at $99.9 \%(\mathrm{ES}(99.9 \%))$ as a risk measure

- - 90th percentile of number of stocks based on ES(99.9\%) as a risk measure

$\longrightarrow$ number of stocks based on Terminal Wealth Standard Deviation (TWSD)

Figure 6: Portfolio size ReQUiREMent For 90\% REDUCtion IN DIVERSifiable Risk. The figure depicts portfolio size recommendations through time and across three different risk measure based on 5-minute. We use expected shortfall as a measure of risk using 5-minute data, and calculate expected shortfall values at 95\%, 99\% and 99.9\% levels. Portfolio size recommendations based on expected shortfall with extreme losses are generally smaller. To obtain the results we repeat the analysis every month using one year of past data. 
Table 1: Conditional correlation coefficients. The table shows average unconditional correlations for each of; the full sample and the pre-crisis period prior to July 2007 and the period thereafter, and conditional correlation coefficients for the three quantiles for the same subsamples.

\begin{tabular}{|c|c|c|c|}
\hline \multirow[t]{2}{*}{ Correlation } & Full period & 01.2003-06.2007 & 07.2007-12.2011 \\
\hline & \multicolumn{3}{|c|}{ Unconditional correlation, $\rho_{i}$} \\
\hline \multirow[t]{2}{*}{$\begin{array}{l}\text { Average } \\
\text { [min; max] } \\
\text { IQR }\end{array}$} & $\begin{array}{c}0.3278 \\
{[0.0716 ; 0.7718]} \\
{[0.2784 ; 0.3725]}\end{array}$ & $\begin{array}{c}0.1899 \\
{[0.0111 ; 0.7041]} \\
{[0.1346 ; 0.2354]}\end{array}$ & $\begin{array}{c}0.3945 \\
{[0.0752 ; 0.8195]} \\
{[0.3417 ; 0.4428]}\end{array}$ \\
\hline & \multicolumn{3}{|c|}{ Conditional correlation (middle), $\rho_{M i}$} \\
\hline \multirow[t]{2}{*}{$\begin{array}{l}\text { Average } \\
\text { [min; max] } \\
\text { IQR }\end{array}$} & $\begin{array}{c}0.2034 \\
{[0.0767 ; 0.5890]} \\
{[0.1703 ; 0.2292]}\end{array}$ & $\begin{array}{c}0.1393 \\
{[0.0249 ; 0.5459]} \\
{[0.1038 ; 0.1688]}\end{array}$ & $\begin{array}{c}0.2717 \\
{[0.1154 ; 0.6760]} \\
{[0.2298 ; 0.3067]}\end{array}$ \\
\hline & \multicolumn{3}{|c|}{ Conditional correlation ( $5 \%$ lower tail), $\rho_{L i}$} \\
\hline \multirow[t]{2}{*}{$\begin{array}{l}\text { Average } \\
\text { [min; max] } \\
\text { IQR }\end{array}$} & $\begin{array}{c}0.3623 \\
{[0.0131 ; 0.7269]} \\
{[0.3042 ; 0.4224]}\end{array}$ & $\begin{array}{c}0.1364 \\
{[-0.1079 ; 0.5786]} \\
{[0.0875 ; 0.1804]}\end{array}$ & $\begin{array}{c}0.3617 \\
{[-0.0129 ; 0.7452]} \\
{[0.2983 ; 0.4290]}\end{array}$ \\
\hline & \multicolumn{3}{|c|}{ Conditional correlation ( $5 \%$ upper tail), $\rho_{U i}$} \\
\hline $\begin{array}{l}\text { Average } \\
\text { [min; max] } \\
\text { IQR }\end{array}$ & $\begin{array}{c}0.4017 \\
{[-0.0299 ; 0.7656]} \\
{[0.3426 ; 0.4648]}\end{array}$ & $\begin{array}{c}0.1481 \\
{[-0.0984 ; 0.6005]} \\
{[0.0913 ; 0.1986]}\end{array}$ & $\begin{array}{c}0.4056 \\
{[0.0193 ; 0.7695]} \\
{[0.3418 ; 0.4735]}\end{array}$ \\
\hline
\end{tabular}

Estimated using the 5-minute return data of S\&P500 constituents and their interquartile range. Overnight returns have been removed prior to correlation estimation. For assets 1 and 2 with returns $r_{1}$ and $r_{2}$, suppose $Q_{L 1}$ and $Q_{L 2}$ are the $p$ per cent quantiles and $Q_{U 1}$ and $Q_{U 2}$ are the $1-p$ per cent quantiles, defining the lower and upper tails of the bivariate distribution of $r_{1}$ and $r_{2}$. Following Silvapulle and Granger (2001), for any given $t$, we define the conditional returns as $\left(r_{L 1 t}, r_{L 2 t}\right)=\left\{\left(r_{1 t}, r_{2 t}\right) \mid r_{1 t}<Q_{L 1}\right.$ and $\left.r_{2 t}<Q_{L 2}\right\}$, $\left(r_{M 1 t}, r_{M 2 t}\right)=\left\{\left(r_{1 t}, r_{2 t}\right) \mid Q_{L 1} \leq r_{1 t} \leq Q_{U 1}\right.$ and $\left.Q_{L 2} \leq r_{2 t} \leq Q_{U 2}\right\}$ and $\left(r_{U 1 t}, r_{U 2 t}\right)=\left\{\left(r_{1 t}, r_{2 t}\right) \mid r_{1 t}>Q_{U 1}\right.$ and $\left.r_{2 t}>Q_{U 2}\right\}$ and the conditional correlations of these returns are $\rho_{L i}, \rho_{M i}$ and $\rho_{U i}$ respectively. 


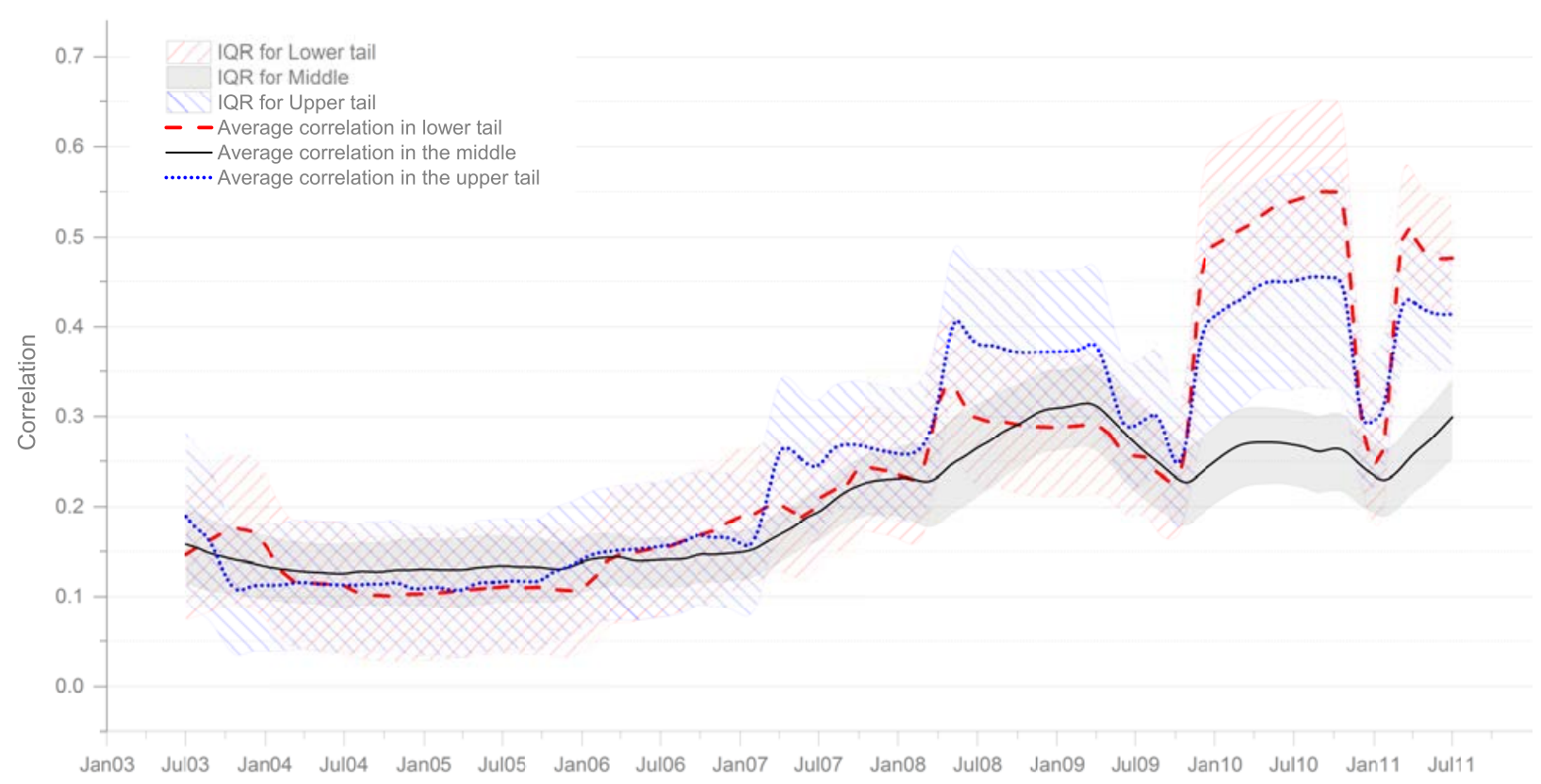

Figure 7: Conditional CORRElation DYNAmics $(p=5 \%)$. The graph depicts average conditional correlations for the 5-minute return data of S\&P500 constituents and their interquartile range. Overnight returns have been removed prior to correlation estimation. For assets 1 and 2 with returns $r_{1}$ and $r_{2}$, suppose $Q_{L 1}$ and $Q_{L 2}$ are the $p$ per cent quantiles and $Q_{U 1}$ and $Q_{U 2}$ are the $1-p$ per cent quantiles, defining the lower and upper tails of the bivariate distribution of $r_{1}$ and $r_{2}$. Following Silvapulle and Granger (2001), for any given $t$, we define the conditional returns as $\left(r_{L 1 t}, r_{L 2 t}\right)=\left\{\left(r_{1 t}, r_{2 t}\right) \mid r_{1 t}<Q_{L 1}\right.$ and $\left.r_{2 t}<Q_{L 2}\right\},\left(r_{M 1 t}, r_{M 2 t}\right)=$ $\left\{\left(r_{1 t}, r_{2 t}\right) \mid Q_{L 1} \leq r_{1 t} \leq Q_{U 1}\right.$ and $\left.Q_{L 2} \leq r_{2 t} \leq Q_{U 2}\right\}$ and $\left(r_{U 1 t}, r_{U 2 t}\right)=\left\{\left(r_{1 t}, r_{2 t}\right) \mid r_{1 t}>Q_{U 1}\right.$ and $\left.r_{2 t}>Q_{U 2}\right\}$ and the conditional correlations of these returns are $\rho_{L i}, \rho_{M i}$ and $\rho_{U i}$ respectively. The figure plots the averages and the interquartile ranges of $\rho_{L i}$ (dashed red line representing the average and right-slanted red pattern area representing the IQR), $\rho_{M i}$ (solid black line and shaded region) and $\rho_{U i}$ (dotted blue line representing and left-slanted blue pattern area). 
Figure 8: CONDITIONAL CORRELATION DYNAMICS BY INDUSTRIES.
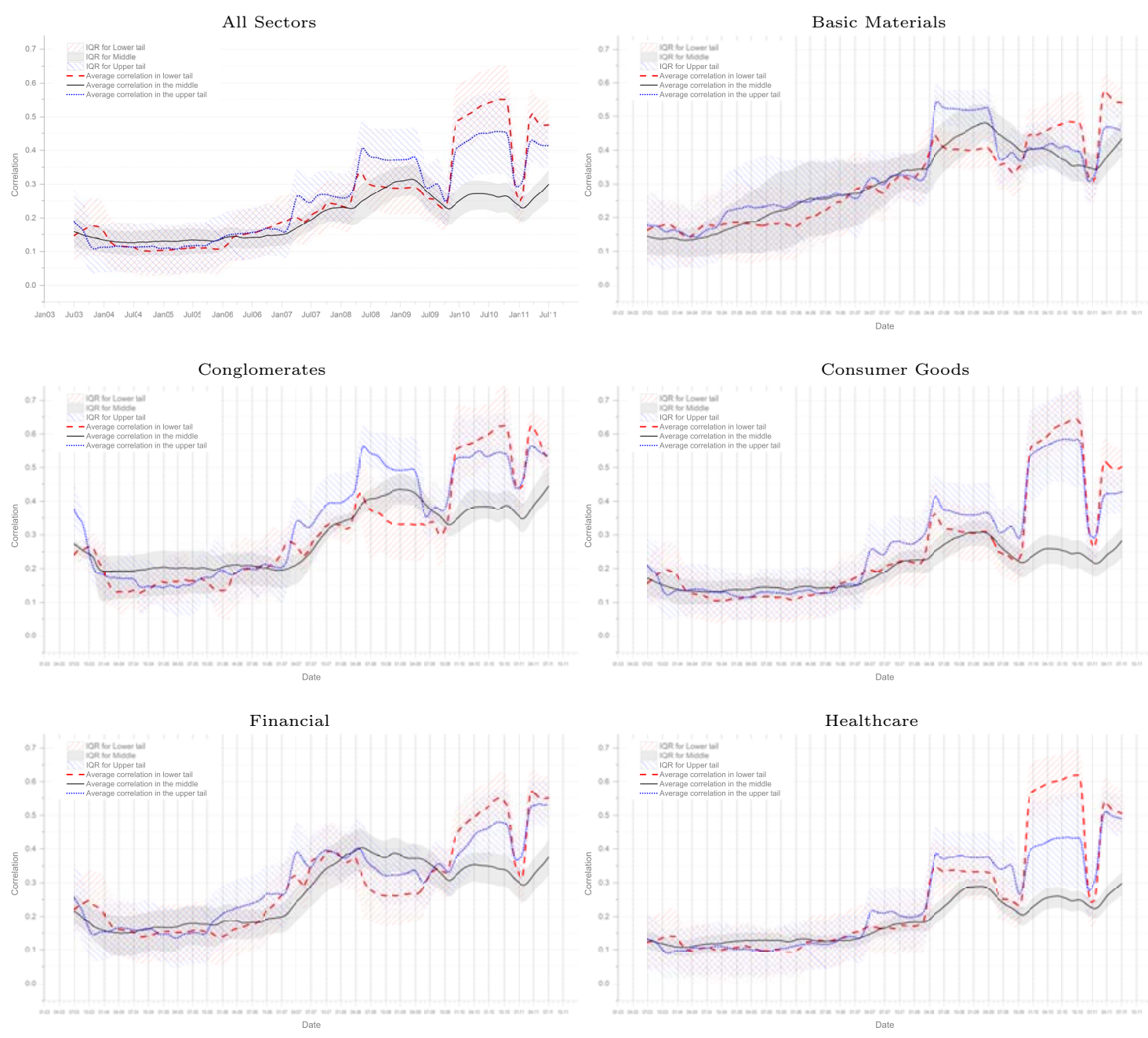
Figure 8: CONTINUED...
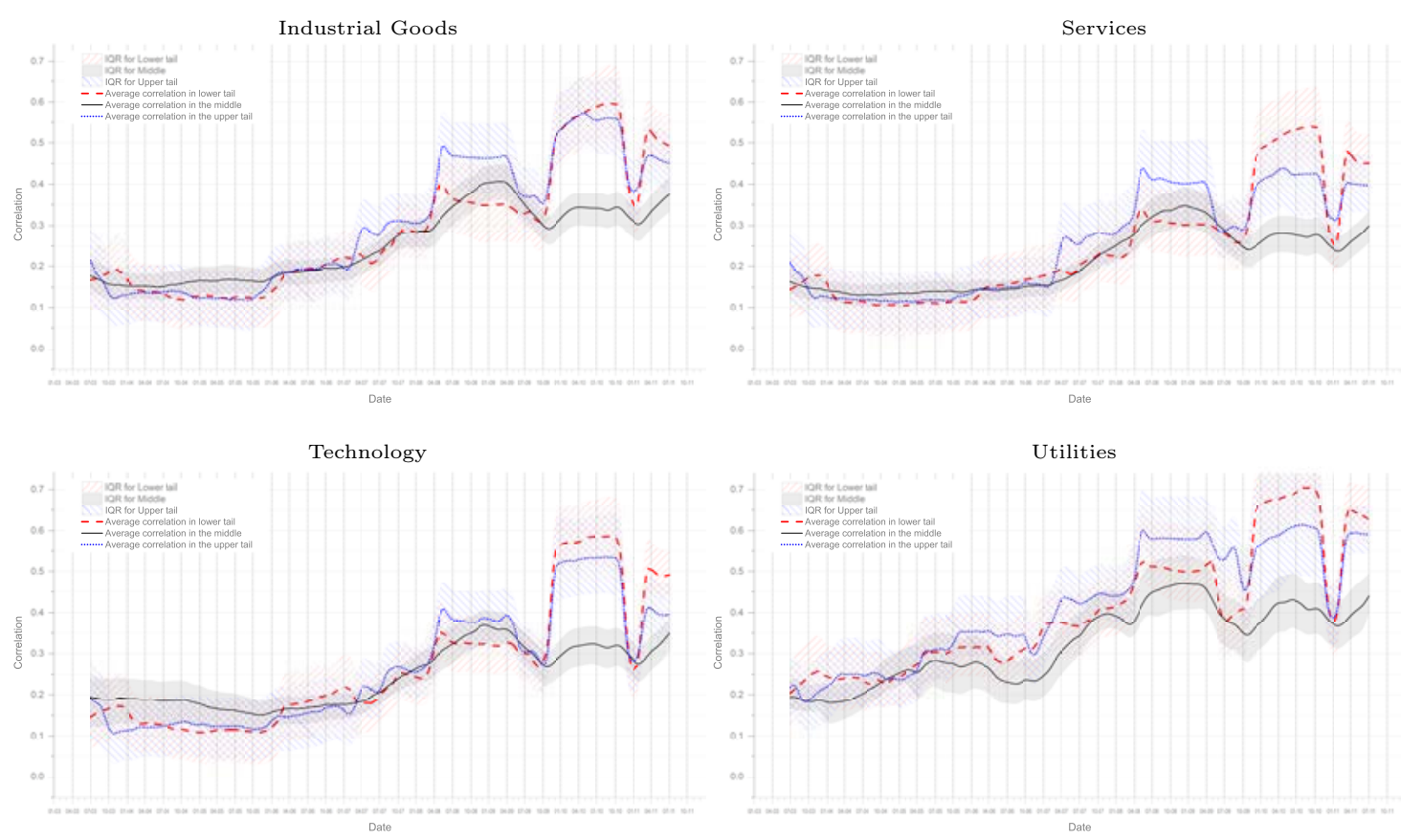


\section{Additional information for web appendix only}

\begin{tabular}{|c|c|c|c|}
\hline RIC Code & Company Name & RIC Code & Company Name \\
\hline A.N & Agilent Technologies Inc & AA.N & Alcoa Inc \\
\hline AAPL.OQ & Apple Inc & ABC.N & AmerisourceBergen Corporation \\
\hline ABT.N & Abbott Laboratories & ACAS.OQ & American Capital Ltd \\
\hline ACE.N & ACE Limited & ACN.N & Accenture plc \\
\hline ADBE.OQ & Adobe Systems Inc & ADI.N & Analog Devices Inc \\
\hline ADM.N & Archer Daniels Midland Company & ADP.OQ & Automatic Data Processing Inc \\
\hline ADSK.OQ & Autodesk Inc & AEE.N & Ameren Corporation \\
\hline AEP.N & American Electric Power Co Inc & AES.N & The AES Corporation \\
\hline AET.N & Aetna Inc & AFL.N & AFLAC Inc \\
\hline AGN.N & Allergan Inc & AIG.N & American International Group Inc \\
\hline AIV.N & Apartment Investment \& Management Co & AIZ.N & Assurant Inc \\
\hline AKAM.Oq & Akamai Technologies Inc & AKS.N & AK Steel Holding Corporation \\
\hline ALL. N & The Allstate Corporation & ALTR.OQ & Altera Corp \\
\hline AM.N & American Greetings Corp & AMAT.OQ & Applied Materials Inc \\
\hline AMCC.OQ & Applied Micro Circuits Corp & AMD.N & Advanced Micro Devices Inc \\
\hline AMGN.OQ & Amgen Inc & AMT.N & American Tower Corporation \\
\hline AMZN.OQ & Amazoncom Inc & AN.N & AutoNation Inc \\
\hline ANF.N & Abercrombie \& Fitch Co & APA.N & Apache Corp \\
\hline APC.N & Anadarko Petroleum Corporation & APD.N & Air Products \& Chemicals Inc \\
\hline APH.N & Amphenol Corporation & APOL.OQ & Apollo Group Inc \\
\hline ARG.N & Airgas Inc & ASH.N & Ashland Inc \\
\hline ATI.N & Allegheny Technologies Inc & AVB.N & Avalonbay Communities Inc \\
\hline AVP.N & Avon Products Inc & AVY.N & Avery Dennison Corporation \\
\hline AXP.N & American Express Company & AZO.N & AutoZone Inc \\
\hline BA.N & Boeing Co & BAC.N & Bank of America Corporation \\
\hline BAX.N & Baxter International Inc & BBBY.OQ & Bed Bath \& Beyond Inc \\
\hline BBT.N & BB\&T Corporation & BBY.N & Best Buy Co Inc \\
\hline BC.N & Brunswick Corporation & BCR.N & CR Bard Inc \\
\hline BDX.N & Becton Dickinson and Company & BEN.N & Franklin Resources Inc \\
\hline BHI.N & Baker Hughes Incorporated & BIIB.OQ & Biogen Idec Inc \\
\hline BK.N & The Bank of New York Mellon Corporation & BLK.N & BlackRock Inc \\
\hline BLL.N & Ball Corporation & BMC.OQ & BMC Software Inc \\
\hline BMS.N & Bemis Company Inc & BMY.N & Bristol-Myers Squibb Company \\
\hline BRCM.OQ & Broadcom Corp & BSX.N & Boston Scientific Corporation \\
\hline BUT.N & Peabody Energy Corp & BWA.N & BorgWarner Inc \\
\hline BXP.N & Boston Properties Inc & C.N & Citigroup Inc \\
\hline CA.OQ & CA Technologies & CAG.N & ConAgra Foods Inc \\
\hline CAH.N & Cardinal Health Inc & CAM.N & Cameron International Corporation \\
\hline CAT.N & Caterpillar Inc & CB.N & The Chubb Corporation \\
\hline CBE.N & Cooper Industries plc & CBG.N & CBRE Group Inc \\
\hline CCE.N & Coca-Cola Enterprises Inc & CCL.N & Carnival Corporation \\
\hline CEG.N & Constellation Energy Group Inc & CELG.OQ & Celgene Corporation \\
\hline CERN.OQ & Cerner Corporation & CHK.N & Chesapeake Energy Corporation \\
\hline CHRQ.OQ & CH Robinson Worldwide Inc & CI.N & Cigna Corp \\
\hline CIEN.OQ & CIENA Corp & CINF.OQ & Cincinnati Financial Corp \\
\hline CL.N & Colgate-Palmolive Co & CLF.N & Cliffs Natural Resources Inc \\
\hline CLX.N & The Clorox Company & CMA.N & Comerica Incorporated \\
\hline CME.OQ & Comcast Corporation & CMI.N & CME Group Inc \\
\hline CMS.N & Cummins Inc & CMSCSA.OQ & CMS Energy Corp \\
\hline CNP.N & CenterPoint Energy Inc & CNX.N & CONSOL Energy Inc \\
\hline COF.N & Capital One Financial Corp & COG.N & Cabot Oil \& Gas Corporation \\
\hline COH.N & Coach Inc & COL.N & Rockwell Collins Inc \\
\hline COP.N & ConocoPhillips & COST.OQ & Costco Wholesale Corporation \\
\hline CPB.N & Campbell Soup Co & CPWR.OQ & Compuware Corporation \\
\hline CR.N & Crane Co & CRM.N & Salesforcecom \\
\hline CSC.N & Computer Sciences Corporation & CSCO.OQ & Cisco Systems Inc \\
\hline CSX.N & CSX Corp & CTAS.OQ & Cintas Corporation \\
\hline CTB.N & Cooper Tire \& Rubber Co & CTL.N & CenturyLink Inc \\
\hline CTSH.OQ & Cognizant Technology Solutions Corporation & CTXS.OQ & Citrix Systems Inc \\
\hline CVC.N & Cablevision Systems Corporation & CVG.N & Convergys Corporation \\
\hline CVH.N & Coventry Health Care Inc & CVS.N & CVS Caremark Corporation \\
\hline CVX.N & Chevron Corporation & D.N & Dominion Resources Inc \\
\hline DD.N & E I du Pont de Nemours and Company & DDR.N & DDR Corp \\
\hline DDS.N & Dillards Inc & DE.N & Deere \& Company \\
\hline
\end{tabular}




\begin{tabular}{|c|c|c|c|}
\hline RIC Code & Company Name & RIC Code & Company Name \\
\hline DELL.OQ & Dell Inc & DF.N & Dean Foods Company \\
\hline DGX.N & Quest Diagnostics Inc & DHI.N & DR Horton Inc \\
\hline DHR.N & Danaher Corp & DIS.N & Walt Disney Co \\
\hline DLTR.OQ & Dollar Tree Inc & DLX.N & Deluxe Corp \\
\hline DNB.N & Dun \& Bradstreet Corp & DNR.N & Denbury Resources Inc \\
\hline DO.N & Diamond Offshore Drilling Inc & DOV.N & Dover Corp \\
\hline DOW.N & The Dow Chemical Company & DRI.N & Darden Restaurants Inc \\
\hline DTE.N & DTE Energy Co & DTV.OQ & DIRECTV Inc \\
\hline DUK.N & Duke Energy Corporation & DV.N & DeVry Inc \\
\hline DVA.N & DaVita Inc & DVN.N & Devon Energy Corporation \\
\hline DYN.N & Dynegy Inc & EA.OQ & Electronic Arts Inc \\
\hline EBAY.OQ & eBay Inc & ECL.N & Ecolab Inc \\
\hline ED.N & Consolidated Edison Inc & EFX.N & Equifax Inc \\
\hline EIX.N & Edison International & EL.N & Estee Lauder Companies Inc \\
\hline EMC.N & EMC Corporation & EMN.N & Eastman Chemical Co \\
\hline EMR.N & Emerson Electric Co & EOG.N & EOG Resources Inc \\
\hline EP.N & El Paso Corp & EQR.N & Equity Residential \\
\hline EQT.N & EQT Corporation & ESRX.OQ & Express Scripts Inc \\
\hline ESV.N & Ensco plc & ETFC.OQ & E_TRADE Financial Corporation \\
\hline ETN.N & Eaton Corporation & ETR.N & Entergy Corporation \\
\hline EW.N & Edwards Lifesciences Corp & EXC.N & Exelon Corporation \\
\hline EXPD.OQ & Expeditors International of Washington Inc & EXPE.OQ & Expedia Inc \\
\hline F.N & Ford Motor Co & FAST.OQ & Fastenal Company \\
\hline FCX.N & Freeport-McMoRan Copper \& Gold Inc & FDO.n & Family Dollar Stores Inc \\
\hline FDX.N & FedEx Corporation & FE.N & FirstEnergy Corp \\
\hline FFIV.OQ & F5 Networks Inc & FHN.N & First Horizon National Corporation \\
\hline FII.N & Federated Investors Inc & FISV.OQ & Fiserv Inc \\
\hline FITB.OQ & Fifth Third Bancorp & FLIR.OQ & FLIR Systems Inc \\
\hline FLR.N & Fluor Corporation & FLS.N & Flowserve Corp \\
\hline FMC.N & FMC Corp & FMCC.OB & Federal Home Loan Mtg \\
\hline FNMA.OB & Fannie Mae & FRX.N & Forest Laboratories Inc \\
\hline FTI.N & FMC Technologies Inc & GAS.N & AGL Resources Inc \\
\hline GCI.N & Gannett Co Inc & GD.N & General Dynamics Corp \\
\hline GE.N & General Electric Company & GGP.N & Gilead Sciences Inc \\
\hline GILD.OQ & General Mills Inc & GIS.N & Corning Inc \\
\hline GLW.N & GameStop Corp & GME.N & Genworth Financial Inc \\
\hline GNW.N & Google Inc & GPC.N & Genuine Parts Company \\
\hline GPS.N & Gap Inc & GR.N & Goodrich Corp \\
\hline GS.N & The Goldman Sachs Group Inc & GT.N & Goodyear Tire \& Rubber Co \\
\hline GWW.N & WW Grainger Inc & HAL.N & Halliburton Company \\
\hline HAR.N & Harman International Industries Inc & HAS.O & Hasbro Inc \\
\hline HBAN.OQ & Huntington Bancshares Incorporated & HCBK.OQ & Hudson City Bancorp Inc \\
\hline HCN.N & Health Care REIT Inc & HCP.N & HCP Inc \\
\hline HD.N & The Home Depot Inc & HIG.N & Hartford Financial Services Group Inc \\
\hline HMA.N & Health Management Associates Inc & HNZ.N & H J Heinz Company \\
\hline HON.N & Honeywell International Inc & HOT.N & Starwood Hotels \& Resorts Worldwide Inc \\
\hline HP.N & Helmerich \& Payne Inc & HPQ.N & Hewlett-Packard Company \\
\hline HRB.N & H\&R Block Inc & HRL.N & Hormel Foods Corp \\
\hline HRS.N & Harris Corp & HSP.N & Hospira Inc \\
\hline HSY.N & Hershey Co & HUM.N & Humana Inc \\
\hline IACI.O & IAC_InterActiveCorp & IBM.N & International Business Machines Corp \\
\hline IFF.N & International Flavors \& Fragrances Inc & IGT.N & International Game Technology \\
\hline INTC.OQ & Intel Corporation & INTU.OQ & Intuit Inc \\
\hline IP.N & International Paper Co & IPG.N & The Interpublic Group of Companies Inc \\
\hline IR.N & Ingersoll-Rand Plc & IRM.N & Iron Mountain Inc \\
\hline ISRG.OQ & Intuitive Surgical Inc & ITT.N & ITT Corporation \\
\hline ITW.N & Illinois Tool Works Inc & JBL.N & Jabil Circuit Inc \\
\hline JCI.N & Johnson Controls Inc & JCP.N & J C Penney Company Inc \\
\hline JDSU.OQ & JDS Uniphase Corporation & JEC.N & Jacobs Engineering Group Inc \\
\hline JNJ.N & Johnson \& Johnson & JNPR.K & Juniper Networks Inc \\
\hline JNS.N & Janus Capital Group Inc & JNY.N & The Jones Group Inc \\
\hline JOY & Joy Global Inc & JPM.N & JPMorgan Chase \& Co \\
\hline JWN.N & Nordstrom Inc & K.N & Kellogg Company \\
\hline KBH.N & KB Home & KEY.N & KeyCorp \\
\hline KFT.N & Kraft Foods Inc & KIM.N & Kimco Realty Corporation \\
\hline KLAC.OQ & KLA-Tencor Corporation & KMB.N & Kimberly-Clark Corporation \\
\hline KMX.N & CarMax Inc & KO.N & The Coca-Cola Company \\
\hline
\end{tabular}




\begin{tabular}{|c|c|c|c|}
\hline RIC Code & Company Name & RIC Code & Company Name \\
\hline KR.N & The Kroger Co & KSS.N & Kohls Corp \\
\hline L.N & Loews Corporation & LEG.N & Leggett \& Platt Incorporated \\
\hline LEH.N & Lehman Brothers & LEN.N & Lennar Corp \\
\hline LH.N & Laboratory Corp of America Holdings & LIFE.OQ & Life Technologies Corporation \\
\hline LIZ.N & Liz Claiborne Inc & LLL.N & L-3 Communications Holdings Inc \\
\hline LLTC.OQ & Linear Technology Corp & LLY.N & Eli Lilly \& Co \\
\hline LM.N & Legg Mason Inc & LMT.N & Lockheed Martin Corporation \\
\hline LNC.N & Lincoln National Corp & LOW.N & Lowes Companies Inc \\
\hline LPX.N & Louisiana-Pacific Corp & LSI.N & LSI Corporation \\
\hline LTD.N & Limited Brands Inc & LUK.N & Leucadia National Corp \\
\hline LUV.N & Southwest Airlines Co & LXK.N & Lexmark International Inc \\
\hline MAR.N & Marriott International Inc & MAS.N & Masco Corporation \\
\hline MAT.O & Mattel Inc & MBI.N & MBIA Inc \\
\hline MCD.N & McDonalds Corp & MCHP.OQ & Microchip Technology Inc \\
\hline MCK.N & McKesson Corporation & MCO.N & Moodys Corp \\
\hline MDP.N & Meredith Corp & MDT.N & Medtronic Inc \\
\hline MET.N & MetLife Inc & MHP.N & The McGraw-Hill Companies Inc \\
\hline MHS.N & Medco Health Solutions Inc & MKC.N & McCormick \& Co Inc \\
\hline MMC.N & Marsh \& McLennan Companies Inc & MMM.N & $3 \mathrm{M}$ Co \\
\hline MO.N & Altria Group Inc & MOLX.OQ & Molex Inc \\
\hline MON.N & Monsanto Co & MOS.N & The Mosaic Company \\
\hline MRK.N & Merck \& Co Inc & MRO.N & Marathon Oil Corporation \\
\hline MS.N & Morgan Stanley & MSFT.OQ & Microsoft Corporation \\
\hline MTB.N & M\&T Bank Corporation & MTG.N & MGIC Investment Corp \\
\hline MTW.N & Manitowoc Co Inc & MU.OQ & Micron Technology Inc \\
\hline MUR.N & Murphy Oil Corporation & MWV.N & MeadWestvaco Corporation \\
\hline MWW & Monster Worldwide Inc & MYL.OQ & Mylan Inc \\
\hline NBL.N & Noble Energy Inc & NBR.N & Nabors Industries Ltd \\
\hline NCR.N & NCR Corp & NDAQ.OQ & Nasdaq OMX Group Inc \\
\hline NE.N & Noble Corp & NEM.N & Newmont Mining Corp \\
\hline NFLX.OQ & Netflix Inc & NFX.N & Newfield Exploration Co \\
\hline NI.N & NiSource Inc & NKE.N & Nike Inc \\
\hline NOC.N & Northrop Grumman Corporation & NOV.N & National Oilwell Varco Inc \\
\hline NRG.N & NRG Energy Inc & NSC.N & Norfolk Southern Corp \\
\hline NTAP.OQ & NetApp Inc & NTRS.OQ & Northern Trust Corporation \\
\hline NU.N & Northeast Utilities & NUE.N & Nucor Corporation \\
\hline NVDA.OQ & NVIDIA Corporation & NVLS.OQ & Novellus Systems Inc \\
\hline NWL.N & Newell Rubbermaid Inc & NWSA.O & News Corp \\
\hline NYT.N & The New York Times Company & ODP.N & Office Depot Inc \\
\hline OI.N & Owens-Illinois Inc & OKE.N & ONEOK Inc \\
\hline OMC.N & Omnicom Group Inc & OMX.N & OfficeMax Incorporated \\
\hline ORCL.OQ & Oracle Corporation & ORLY.OQ & OReilly Automotive Inc \\
\hline OXY.N & Occidental Petroleum Corporation & PAYX.OQ & Paychex Inc \\
\hline PBCT.OQ & Peoples United Financial Inc & PBI.N & Pitney Bowes Inc \\
\hline PCAR.OQ & PACCAR Inc & PCG.N & PG\&E Corp \\
\hline PCL.N & Plum Creek Timber Co Inc & PCLN.OQ & pricelinecom Incorporated \\
\hline PCP.N & Precision Castparts Corp & PDCO.OQ & Patterson Companies Inc \\
\hline PEG.N & Public Service Enterprise Group Inc & PEP.N & Pepsico Inc \\
\hline PFE.N & Pfizer Inc & PFG.N & Principal Financial Group Inc \\
\hline PG.N & Procter \& Gamble Co & PGN.N & Progress Energy Inc \\
\hline PGR.N & Progressive Corp & PH.N & Parker Hannifin Corporation \\
\hline PHM.N & PulteGroup Inc & PKI.N & PerkinElmer Inc \\
\hline PLD.N & Prologis Inc & PLL.N & Pall Corp \\
\hline PMCS.OQ & PMC-Sierra Inc & PMTC.OQ & Parametric Technology Corporation \\
\hline PNC.N & PNC Financial Services Group Inc & PNW.N & Pinnacle West Capital Corporation \\
\hline POM.N & Pepco Holdings Inc & PPG.N & PPG Industries Inc \\
\hline PPL.N & PPL Corporation & PRGO.OQ & Perrigo Co \\
\hline PRU.N & Prudential Financial Inc & PSA.N & Public Storage \\
\hline PWER.OQ & Power-One Inc & PWR.N & Quanta Services Inc \\
\hline PX.N & Praxair Inc & PXD.N & Pioneer Natural Resources Co \\
\hline QCOM.OQ & QUALCOMM Incorporated & QLGC.OQ & QLogic Corp \\
\hline R.N & Ryder System Inc & RAI.N & Reynolds American Inc \\
\hline RDC.N & Rowan Companies Inc & RF.N & Regions Financial Corp \\
\hline RHI.N & Robert Half International Inc & RIG.N & Transocean Ltd \\
\hline RL.N & Ralph Lauren Corporation & ROK.N & Rockwell Automation Inc \\
\hline ROP.N & Roper Industries Inc & ROST.OQ & Ross Stores Inc \\
\hline RRC.N & Range Resources Corporation & RRD.OQ & RR Donnelley \& Sons Company \\
\hline
\end{tabular}




\begin{tabular}{|c|c|c|c|}
\hline RIC Code & Company Name & RIC Code & Company Name \\
\hline RSG.N & Republic Services Inc & RSH.N & RadioShack Corp \\
\hline RTN.N & Raytheon Co & S.N & Sprint Nextel Corp \\
\hline SANM.OQ & Sanmina-SCI Corp & SBUX.OQ & Starbucks Corporation \\
\hline SCG.N & SCANA Corp & SE.N & Spectra Energy Corp \\
\hline SEE.N & Sealed Air Corporation & SHLD.OQ & Sears Holdings Corporation \\
\hline SHW.N & The Sherwin-Williams Company & SIAL.OQ & Sigma-Aldrich Corporation \\
\hline SJM.N & The J M Smucker Company & SLB.N & Schlumberger Limited \\
\hline SLE.N & Sara Lee Corp & SLM.O & SLM Corporation \\
\hline SNA.N & Snap-on Inc & SNDK.OQ & SanDisk Corp \\
\hline SNV.N & Synovus Financial Corp & SO.N & Southern Company \\
\hline SPG.N & Simon Property Group Inc & SPLS.OQ & Staples Inc \\
\hline SRCL.OQ & Stericycle Inc & SRE.N & Sempra Energy \\
\hline SSP.N & The E W Scripps Company & STI.N & SunTrust Banks Inc \\
\hline STJ.N & St Jude Medical Inc & STR.N & Questar Corporation \\
\hline STT.N & State Street Corp & STZ.N & Constellation Brands Inc \\
\hline SUN.N & Sunoco Inc & SVU.N & SUPERVALU Inc \\
\hline SWK.N & Stanley Black \& Decker Inc & SWN.N & Southwestern Energy Co \\
\hline SWY.N & Safeway Inc & SYK.N & Stryker Corp \\
\hline SYMC.OQ & Symantec Corporation & SYY.N & Sysco Corp \\
\hline T.N & AT\&T Inc & TAP.N & Molson Coors Brewing Company \\
\hline TE.N & TECO Energy Inc & TER.N & Teradyne Inc \\
\hline TEX.N & Terex Corp & TGT.N & Target Corp \\
\hline THC.N & Tenet Healthcare Corp & TIE.N & Titanium Metals Corporation \\
\hline TIF.N & Tiffany \& Co & TIN.N & Temple-Inland Inc \\
\hline TJX.N & The TJX Companies Inc & TLAB.OQ & Tellabs Inc \\
\hline TMK.N & Torchmark Corp & TMO.N & Thermo Fisher Scientific Inc \\
\hline TNB.N & Thomas \& Betts Corp & TROW.OQ & T Rowe Price Group Inc \\
\hline TSN.N & Tyson Foods Inc & TSO.N & Tesoro Corporation \\
\hline TSS.N & Total System Services Inc & TUP.N & Tupperware Brands Corporation \\
\hline TWX.N & Time Warner Inc & TXN.N & Texas Instruments Inc \\
\hline TXT.N & Textron Inc & TYC.N & Tyco International Ltd \\
\hline UIS.N & Unisys Corporation & UNH.N & Unitedhealth Group Inc \\
\hline UNM.N & Unum Group & UNP.N & Union Pacific Corporation \\
\hline UPS.N & United Parcel Service Inc & URBN.OQ & Urban Outfitters Inc \\
\hline USB.N & US Bancorp & UTX.N & United Technologies Corp \\
\hline VAR.N & Varian Medical Systems Inc & VFC.N & VF Corporation \\
\hline VLO.N & Valero Energy Corporation & VMC.N & Vulcan Materials Company \\
\hline VNO.N & Vornado Realty Trust & VRSN.OQ & VeriSign Inc \\
\hline VTR.N & Ventas Inc & VZ.N & Verizon Communications Inc \\
\hline WAG.N & Walgreen Co & WAT.N & Waters Corp \\
\hline WDC.N & Western Digital Corp & WEC.N & Wisconsin Energy Corp \\
\hline WFC.N & Wells Fargo \& Company & WFR.N & MEMC Electronic Materials Inc \\
\hline WFT.N & Weatherford International Ltd & WHR.N & Whirlpool Corp \\
\hline WLP.N & WellPoint Inc & WM.N & Waste Management Inc \\
\hline WMB.N & Williams Companies Inc & WMT.N & Wal-Mart Stores Inc \\
\hline WOR.N & Worthington Industries Inc & WPI.N & Watson Pharmaceuticals Inc \\
\hline WPO.N & The Washington Post Company & WY.N & Weyerhaeuser Co \\
\hline WYNN.OQ & Wynn Resorts Ltd & X.N & United States Steel Corp \\
\hline XEL.N & Xcel Energy Inc & XL.N & XL Group plc \\
\hline XLNX.OQ & Xilinx Inc & XOM.N & Exxon Mobil Corporation \\
\hline XRAY.OQ & DENTSPLY International Inc & XRX.N & Xerox Corp \\
\hline YHOO.OQ & Yahoo! Inc & YUM.N & Yum! Brands Inc \\
\hline ZION.OQ & Zions Bancorp & ZMH.N & Zimmer Holdings Inc \\
\hline
\end{tabular}




\section{School of Economics and Finance Discussion Papers}

2013-20 Towards a Diagnostic Approach to Climate Adaptation for Fisheries, P. Leith, E. Ogier, G. Pecl, E. Hoshino, J. Davidson, M. Haward

2013-19 Equity Portfolio Diversification with High Frequency Data, Vitali Alexeev and Mardi Dungey

2013-18 Measuring the Performance of Hedge Funds Using Two-Stage Peer Group Benchmarks, Marco Wilkens, Juan Yao, Nagaratnam Jeyasreedharan and Patrick Oehler

2013-17 What Australian Investors Need to Know to Diversify their Portfolios, Vitali Alexeev and Francis Tapon

2013-16 Equity Portfolio Diversification: How Many Stocks are Enough? Evidence from Five Developed Markets, Vitali Alexeev and Francis Tapon

2013-15 Equity market Contagion during the Global Financial Crisis: Evidence from the World’s Eight Largest Economies, Mardi Dungey and Dinesh Gajurel

2013-14 A Survey of Research into Broker Identity and Limit Order Book, Thu Phuong Pham and P Joakim Westerholm

2013-13 Broker ID Transparency and Price Impact of Trades: Evidence from the Korean Exchange, Thu Phuong Pham

2013-12 An International Trend in Market Design: Endogenous Effects of Limit Order Book Transparency on Volatility, Spreads, depth and Volume, Thu Phuong Pham and P Joakim Westerholm

2013-11 On the Impact of the Global Financial Crisis on the Euro Area, Xiaoli He, Jan PAM Jacobs, Gerald H Kuper and Jenny E Ligthart

2013-10 International Transmissions to Australia: The Roles of the US and Euro Area, Mardi Dungey, Denise Osborn and Mala Raghavan

2013-09 Are Per Capita $\mathrm{CO}_{2}$ Emissions Increasing Among OECD Countries? A Test of Trends and Breaks, Satoshi Yamazaki, Jing Tian and Firmin Doko Tchatoka

2013-08 Commodity Prices and BRIC and G3 Liquidity: A SFAVEC Approach, Ronald A Ratti and Joaquin L Vespignani

2013-07 Chinese Resource Demand and the Natural Resource Supplier Mardi Dungy, Renée Fry-McKibbin and Verity Linehan

2013-06 Not All International Monetary Shocks are Alike for the Japanese Economy, Joaquin L Vespignani and Ronald A Ratti

2013-05 On Bootstrap Validity for Specification Tests with Weak Instruments, Firmin Doko Tchatoka

2013-04 Chinese Monetary Expansion and the US Economy, Joaquin L Vespignani and Ronald A Ratti

2013-03 International Monetary Transmission to the Euro Area: Evidence from the US, Japan and China, Joaquin L Vespignani and Ronald A Ratti

2013-02 The impact of jumps and thin trading on realized hedge ratios? Mardi Dungey, Olan T. Henry, Lyudmyla Hvozdyk

2013-01 Why crude oil prices are high when global activity is weak?, Ronald A Rattia and Joaquin L Vespignani

Copies of the above mentioned papers and a list of previous years' papers are available from our home site at http://www.utas.edu.au/economics-finance/research/ 